\title{
A System of Generalized Mixed Equilibrium Problems, Maximal Monotone Operators, and Fixed Point Problems with Application to Optimization Problems
}

\author{
Pongsakorn Sunthrayuth ${ }^{1,2}$ and Poom Kumam ${ }^{1,2}$ \\ ${ }^{1}$ Department of Mathematics, Faculty of Science, King Mongkut's University of Technology Thonburi \\ (KMUTT), Bangmod, Bangkok 10140, Thailand \\ ${ }^{2}$ Centre of Excellence in Mathematics, CHE, Si Ayutthaya Road, Bangkok 10400, Thailand \\ Correspondence should be addressed to Poom Kumam, poom.kum@kmutt.ac.th
}

Received 30 August 2011; Accepted 1 November 2011

Academic Editor: Muhammad Aslam Noor

Copyright (c) 2012 P. Sunthrayuth and P. Kumam. This is an open access article distributed under the Creative Commons Attribution License, which permits unrestricted use, distribution, and reproduction in any medium, provided the original work is properly cited.

\begin{abstract}
We introduce a new iterative algorithm for finding a common element of the set of solutions of a system of generalized mixed equilibrium problems, zero set of the sum of a maximal monotone operators and inverse-strongly monotone mappings, and the set of common fixed points of an infinite family of nonexpansive mappings with infinite real number. Furthermore, we prove under some mild conditions that the proposed iterative algorithm converges strongly to a common element of the above four sets, which is a solution of the optimization problem related to a strongly positive bounded linear operator. The results presented in the paper improve and extend the recent ones announced by many others.
\end{abstract}

\section{Introduction}

Throughout this paper, we denoted by $\mathbb{N}$ and $\mathbb{R}^{+}$the set of all positive integers and all positive real numbers, respectively. We always assume that $H$ is a real Hilbert space with inner product $\langle\cdot, \cdot\rangle$ and norm $\|\cdot\|$, respectively, and $C$ is a nonempty closed convex subset of $H$. Let $\varphi: C \rightarrow \mathbb{R}$ be a real-valued function, $\Theta: C \times C \rightarrow \mathbb{R}$ be an equilibrium bifunction, and $\Psi, \Phi: C \rightarrow H$ be two nonlinear mappings. The generalized mixed equilibrium problem with perturbed mapping is to find $x^{*} \in C$ such that

$$
\Theta\left(x^{*}, y\right)+\varphi(y)-\varphi\left(x^{*}\right)+\left\langle(\Psi+\Phi) x^{*}, y-x^{*}\right\rangle \geq 0, \quad \forall y \in C
$$


The set of solutions of the problem (1.1) is denoted by $\operatorname{GMEP}(\Theta, \varphi, \Psi+\Phi)$. As special cases of the problem (1.1), we have the following.

(1) If $\Phi=0$, then the problem (1.1) reduces to the generalized mixed equilibrium problem of finding $x^{*} \in C$ such that

$$
\Theta\left(x^{*}, y\right)+\varphi(y)-\varphi\left(x^{*}\right)+\left\langle\Psi x^{*}, y-x^{*}\right\rangle \geq 0, \quad \forall y \in C,
$$

which was introduced and studied by Peng and Yao [1]. The set of solutions of the problem (1.2) is denoted by $\operatorname{GMEP}(\Theta, \varphi, \Psi)$.

(2) If $\Psi=\Phi=0$, then the problem (1.1) reduces to the mixed equilibrium problem of finding $x^{*} \in C$ such that

$$
\Theta\left(x^{*}, y\right)+\varphi(y)-\varphi\left(x^{*}\right) \geq 0, \quad \forall y \in C
$$

which was consider by Ceng and Yao [2]. The set of solutions of the problem (1.3) is denoted by $\operatorname{MEP}(\Theta)$.

(3) If $\Phi=\varphi=0$, then the problem (1.1) reduces to the generalized equilibrium problem of finding $x^{*} \in C$ such that

$$
\Theta\left(x^{*}, y\right)+\left\langle\Psi x^{*}, y-x^{*}\right\rangle \geq 0, \quad \forall y \in C,
$$

which was consider by S. Takahashi and W. Takahashi [3]. The set of solutions of the problem (1.4) is denoted by $\operatorname{GEP}(\Theta, \Psi)$.

(4) If $\Psi=\Phi=\varphi=0$, then the problem (1.1) reduces to the equilibrium problem of finding $x^{*} \in C$ such that

$$
\Theta\left(x^{*}, y\right) \geq 0, \quad \forall y \in C
$$

The set of solutions of the problem (1.5) is denoted by $\operatorname{EP}(\Theta)$.

(5) If $\Theta=\Phi=\varphi=0$, then the problem (1.1) reduces to the classical variational inequality problem of finding $x^{*} \in C$ such that

$$
\left\langle\Psi x^{*}, y-x^{*}\right\rangle \geq 0, \quad \forall y \in C
$$

The set of solutions of the problem (1.6) is denoted by $\operatorname{VI}(C, \Psi)$. It is known that $x^{*} \in C$ is a solution of the problem (1.6) if and only if $x^{*}$ is a fixed point of the mapping $P_{C}(I-\lambda \Psi)$, where $\lambda>0$ is a constant and $I$ is the identity mapping. 
The generalized mixed equilibrium problems with perturbation is very general in the sense that it includes fixed point problems, optimization problems, variational inequality problems, Nash equilibrium problems, and equilibrium problems as special cases (see, e.g., $[4,5]$ ). Numerous problems in physics, optimization, and economics reduce to find a solution of problem (1.2). Several methods have been proposed to solve the fixed point problems, variational inequality problems, and equilibrium problems in the literature (see, e.g., [6-34]).

Let $A$ be a strongly positive bounded linear operator on $H$; that is, there exists a constant $\bar{\gamma}>0$ such that

$$
\langle A x, x\rangle \geq \bar{\gamma}\|x\|^{2}, \quad \forall x \in H
$$

Recall that a mapping $f: C \rightarrow C$ is said to be contractive if there exists a constant $\alpha \in(0,1)$ such that

$$
\|f(x)-f(y)\| \leq \alpha\|x-y\|, \quad \forall x, y \in C
$$

A mapping $T: C \rightarrow C$ is said to be

(1) nonexpansive if

$$
\|T x-T y\| \leq\|x-y\|, \quad \forall x, y \in C
$$

(2) firmly nonexpansive if

$$
\|T x-T y\|^{2} \leq\langle T x-T y, x-y\rangle, \quad \forall x, y \in C,
$$

(3) $k$-strictly pseudocontractive if there exists a constant $k \in[0,1)$ such that

$$
\|T x-T y\|^{2} \leq\|x-y\|^{2}+k\|(I-T) x-(I-T) y\|^{2}, \quad \forall x, y \in C .
$$

We denote by $F(T)$ the set of fixed points of $T$, that is, $F(T)=\{x \in C: x=T x\}$. mentioned.

Recall the following definitions of a nonlinear mapping $B: C \rightarrow H$; the following is Definition 1.1. The nonlinear mapping $B: C \rightarrow H$ is said to be

(i) monotone if

$$
\langle B x-B y, x-y\rangle \geq 0, \quad \forall x, y \in C,
$$

(ii) $\beta$-strongly monotone if there exists a constant $\beta>0$ such that

$$
\langle B x-B y, x-y\rangle \geq \beta\|x-y\|^{2}, \quad \forall x, y \in C,
$$


(iii) $v$-inverse-strongly monotone if there exists a constant $v>0$ such that

$$
\langle B x-B y, x-y\rangle \geq v\|B x-B y\|^{2}, \quad \forall x, y \in C
$$

Let $W: H \rightarrow 2^{H}$ be a set-valued mapping. The set $D(W)$ defined by $D(W)=\{x \in H$ : $W x \neq \emptyset\}$ is said to be the domain of $W$. The set $R(W)$ defined by $R(W)=\bigcup_{x \in H} W x$ is said to be the range of $W$. The set $G(W)$ defined by $G(W)=\{(x, y) \in H \times H: x \in D(W), y \in R(W)\}$ is said to be the graph of $W$.

Recall that $W$ is said to be monotone if

$$
\langle x-y, f-g\rangle>0, \quad \forall(x, f),(y, g) \in G(W) .
$$

$W$ is said to be maximal monotone if it is not properly contained in any other monotone operator. Equivalently, $W$ is maximal monotone if $R(I+r W)=H$ for all $r>0$. For a maximal monotone operator $M$ on $H$ and $r>0$, we may define the single-valued resolvent $J_{r}=(I+r W)^{-1}: H \rightarrow D(W)$. It is known that $J_{r}$ is firmly nonexpansive $W^{-1}(0)=F\left(J_{r}\right)$, where $F\left(J_{r}\right)$ denotes the fixed point set of $J_{r}$.

We discuss the following algorithms for solving the solutions of variational inequality problems and fixed point problems for a nonexpansive mapping (see, e.g., [29, 35-43]).

In 2010, Chantarangsi et al. [44] introduced a new viscosity hybrid steepest descent method for solving the generalized mixed equilibrium problems (1.2), variational inequality problems, and fixed point problems of nonexpansive mappings in a real Hilbert space. More precisely, they proved the following theorem.

\section{Theorem CCK [see [44]]}

Let $C$ be a nonempty closed and convex subset of a real Hilbert space $H$. Let $\Theta_{1}, \Theta_{2}$ be two bifunctions satisfying condition (H1)-(H5), let $\Psi_{1}, \Psi_{2}$ be $\xi$-inverse-strongly monotone mapping and $\beta$-inverse-strongly monotone mapping, respectively, and let $T: C \rightarrow C$ be a nonexpansive mapping. Let $B$ be an $\omega$-Lipschitz continuous and relaxed $(v, v)$ cocoercive mapping, $f: C \rightarrow C$ a contraction mapping with coefficient $\alpha \in(0,1)$, and $A$ a strongly positive linear bounded self-adjoint operator with coefficient $\bar{\gamma}>0$ and $0<\gamma<\bar{\gamma} / \alpha$. Suppose that $\Omega:=F(T) \cap \operatorname{GMEP}\left(\Theta_{1}, \varphi, \Psi_{1}\right) \cap \operatorname{GMEP}\left(\Theta_{2}, \varphi, \Psi_{2}\right) \cap \operatorname{VI}(C, B)$. Let $\left\{z_{n}\right\},\left\{u_{n}\right\},\left\{y_{n}\right\}$, and $\left\{x_{n}\right\}$ be generated by

$$
\begin{gathered}
u_{n}=V_{\left.r_{n}, \varphi_{2}\right)}^{\left(\Theta_{2}\right)}\left(x_{n}-r_{n} \Psi_{2} x_{n}\right), \\
v_{n}=V_{\mu_{n}}^{\left(\Theta_{1}, \varphi_{1}\right)}\left(u_{n}-\mu_{n} \Psi_{1} u_{n}\right), \\
z_{n}=P_{C}\left(v_{n}-\alpha_{n} B T v_{n}\right), \\
y_{n}=\epsilon_{n} \gamma f\left(x_{n}\right)+\beta_{n} x_{n}+\left(\left(1-\beta_{n}\right) I-\epsilon_{n} A\right) z_{n}, \\
x_{n+1}=\gamma_{n} x_{n}+\left(1-\gamma_{n}\right) y_{n}, \quad \forall n \geq 1,
\end{gathered}
$$

where $\left\{\gamma_{n}\right\} \subset[a, b] \subset[0,2 \xi],\left\{s_{n}\right\} \subset[c, d] \subset[0,2 \beta],\left\{\gamma_{n}\right\} \subset[h, j] \subset(0,1),\left\{\gamma_{n}\right\},\left\{\epsilon_{n}\right\}$, and $\left\{\beta_{n}\right\}$ are three sequences in $(0,1)$ satisfying the following conditions: 
(C1) $\lim _{n \rightarrow \infty} \epsilon_{n}=0$ and $\sum_{n=1}^{\infty} \epsilon_{n}=\infty$,

(C2) $0<\liminf _{n \rightarrow \infty} \beta_{n} \leq \lim \sup _{n \rightarrow \infty} \beta_{n}<1$,

(C3) $0<\liminf _{n \rightarrow \infty} \mu_{n} \leq \lim \sup _{n \rightarrow \infty} \mu_{n}<2 \beta$ and $\lim _{n \rightarrow \infty}\left|\mu_{n+1}-\mu_{n}\right|=0$,

(C4) $0<\liminf _{n \rightarrow \infty} r_{n} \leq \limsup _{n \rightarrow \infty} r_{n}<2 \xi$ and $\lim _{n \rightarrow \infty}\left|r_{n+1}-r_{n}\right|=0$,

(C5) $\left\{\alpha_{n}\right\} \subset[e, g] \subset\left(0,2\left(v-v \omega^{2}\right) / \omega^{2}\right), v>v \omega^{2}$ and $\lim _{n \rightarrow \infty}\left|\alpha_{n+1}-\alpha_{n}\right|=0$.

Then, $\left\{x_{n}\right\}$ converges strongly to $x^{*}=P_{\Omega}(\gamma f+(I-A))\left(x^{*}\right)$.

Very recently, Yu and Liang [45] proved the following convergence theorem of finding a common element in the fixed point set of a strict pseudocontraction and in the zero set of a nonlinear mapping which is the sum of a maximal monotone operator and inverse strongly monotone mapping in a real Hilbert space.

\section{Theorem YL [see [45]]}

Let $H$ be a real Hilbert space and $C$ a nonempty close and convex subset of $H$. Let $W_{1}$ : $H \rightarrow 2^{H}$ and $W_{2}: H \rightarrow 2^{H}$ be two maximal monotone operators such that $D\left(W_{1}\right) \subset C$ and $D\left(W_{2}\right) \subset C$, respectively. Let $S: C \rightarrow C$ be a $k$-strict pseudocontraction mapping, $A: C \rightarrow H$ an $\alpha$-inverse-strongly monotone mapping, and $B: C \rightarrow H$ an $\beta$-inverse-strongly monotone mapping. Assume that $\Omega:=F(S) \cap\left(A+W_{1}\right)^{-1}(0) \cap\left(B+W_{2}\right)^{-1}(0) \neq \emptyset$. Let $\left\{x_{n}\right\}$ be a sequence generated by

$$
\begin{gathered}
x_{1} \in C, \\
y_{n}=J_{t_{n}}\left(x_{n}-t_{n} B x_{n}\right), \\
x_{n+1}=\alpha_{n} u+\beta_{n} x_{n}+\gamma_{n}\left[\delta_{n} J_{s_{n}}\left(y_{n}-s_{n} A y_{n}\right)+\left(1-\delta_{n}\right) J_{s_{n}}\left(y_{n}-s_{n} A y_{n}\right)\right], \quad \forall n \geq 1,
\end{gathered}
$$

where $u \in C$ is a fixed element, $J_{s_{n}}=\left(I+s_{n} W_{1}\right)^{-1}, J_{t_{n}}=\left(I+t_{n} W_{2}\right)^{-1},\left\{s_{n}\right\}$ is a sequence in $(0,2 \alpha),\left\{t_{n}\right\}$ is a sequence in $(0,2 \beta)$, and $\left\{\alpha_{n}\right\},\left\{\beta_{n}\right\},\left\{\gamma_{n}\right\}$, and $\left\{\delta_{n}\right\}$ are sequences in $[0,1]$ satisfying the following conditions:

$$
\begin{aligned}
& \text { (C1) } \lim _{n \rightarrow \infty} \alpha_{n}=0 \text { and } \sum_{n=1}^{\infty} \alpha_{n}=\infty, \\
& \text { (C2) } 0<\liminf _{n \rightarrow \infty} \beta_{n} \leq \lim \sup _{n \rightarrow \infty} \beta_{n}<1, \\
& \text { (C3) } 0<a \leq s_{n} \leq b<2 \alpha \text { and } \lim _{n \rightarrow \infty}\left(s_{n+1}-s_{n}\right)=0, \\
& \text { (C4) } 0<c \leq t_{n} \leq d<2 \beta \text { and } \lim _{n \rightarrow \infty}\left(t_{n+1}-t_{n}\right)=0, \\
& \text { (C5) } 0<c \leq k \leq \delta_{n}<e<1 \text { and } \lim _{n \rightarrow \infty}\left(\delta_{n+1}-\delta_{n}\right)=0 \text {. }
\end{aligned}
$$

Then, the sequence $\left\{x_{n}\right\}$ converges strongly to $x^{*}=P_{\Omega} x^{*}$.

On the other hand, the following optimization problem has been studied extensively by many authors:

$$
\min _{x \in \bar{\Omega}} \frac{\mu}{2}\langle A x, x\rangle+\frac{1}{2}\|x-u\|^{2}-h(x)
$$

where $\bar{\Omega}=\bigcap_{n=1}^{\infty} C_{n}, C_{1}, C_{2}, \ldots$ are infinitely many closed convex subsets of $H$ such that $\bigcap_{n=1}^{\infty} C_{n} \neq \emptyset, u \in H, \mu \geq 0$ is a real number, $A$ is a strongly positive linear bounded operator 
on $H$, and $h$ is a potential function for $\gamma f$ (i.e., $h^{\prime}(x)=\gamma f(x)$ for all $x \in H$ ). This kind of optimization problem has been studied extensively by many authors (see, e.g., [5, 46-52]) for when $\bar{\Omega}=\bigcap_{n=1}^{\infty} C_{n}$ and $h(x)=\langle x, b\rangle$, where $b$ is a given point in $H$.

The following questions naturally arise in connection with above the results.

Question 1. Could we weaken the control conditions of Theorems CCK and YL in (C3) and (C4)?

Question 2. Can Theorem YL be extended to finding a common element of the set of solutions of a system generalized mixed equilibrium problems and the set of common fixed points of infinite family of nonexpansive mappings?

The purpose of this paper is to give the affirmative answers to these questions mentioned above. Motivated by the iterative process (1.16) and (1.17), we introduce a new iterative algorithm (3.2) below, for finding a common element of the set of solutions of a system of generalized mixed equilibrium problems, zero set of the sum of a maximal monotone operators and inverse-strongly monotone mappings, and the set of common fixed points of an infinite family of nonexpansive mappings with infinite real number. Then, we prove the strong convergence theorem of these iterative process in a real Hilbert space. The results presented in the paper improve and extend the recent ones announced by many others.

\section{Preliminaries}

Definition 2.1 (see [53]). Let $C$ be a nonempty convex subset of a real Hilbert space $H$. Let $T_{i}$, $i=1,2, \ldots$, be mappings of $C$ into itself. For each $j=1,2, \ldots$, let $\alpha_{j}=\left(\alpha_{1}^{j}, \alpha_{2}^{j}, \alpha_{3}^{j}\right) \in I \times I \times I$, where $I=[0,1]$ and $\alpha_{1}^{j}+\alpha_{2}^{j}+\alpha_{3}^{j}=1$. For every $n \in \mathbb{N}$, we define the mapping $S_{n}: C \rightarrow C$ as follows:

$$
\begin{gathered}
U_{n, n+1}=I, \\
U_{n, n}=\alpha_{1}^{n} T_{n} U_{n, n+1}+\alpha_{2}^{n} U_{n, n+1}+\alpha_{3}^{n} I, \\
U_{n, n-1}=\alpha_{1}^{n-1} T_{n-1} U_{n, n}+\alpha_{2}^{n-1} U_{n, n}+\alpha_{3}^{n-1} I, \\
\vdots \\
U_{n, k+1}=\alpha_{1}^{k+1} T_{k+1} U_{n, k+2}+\alpha_{2}^{k+1} U_{n, k+2}+\alpha_{3}^{k+1} I, \\
U_{n, k}=\alpha_{1}^{k} T_{k} U_{n, k+1}+\alpha_{2}^{k} U_{n, k+1}+\alpha_{3}^{k} I, \\
\vdots \\
U_{n, 2}=\alpha_{1}^{2} T_{2} U_{n, 3}+\alpha_{2}^{2} U_{n, 3}+\alpha_{3}^{2} I, \\
S_{n}=U_{n, 1}=\alpha_{1}^{1} T_{1} U_{n, 2}+\alpha_{2}^{1} U_{n, 2}+\alpha_{3}^{1} I .
\end{gathered}
$$


Such a mapping $S_{n}$ is nonexpansive from $C$ into itself, and it is called $S$-mapping generated by $T_{n}, T_{n-1}, \ldots, T_{1}$ and $\alpha_{n}, \alpha_{n-1}, \ldots, \alpha_{1}$.

Lemma 2.2 (see [53]). Let $C$ be a nonempty closed convex subset of a real Hilbert space $H$. Let $\left\{T_{i}\right\}_{i=1}^{\infty}$ be nonexpansive mappings of $C$ into itself with $F\left(T_{i}\right) \neq \emptyset$ and let $\alpha_{j}=\left(\alpha_{1}^{j}, \alpha_{2}^{j}, \alpha_{3}^{j}\right) \in I \times I \times I$, where $I=[0,1], \alpha_{1}^{j}+\alpha_{2}^{j}+\alpha_{3}^{j}=1, \alpha_{1}^{j}+\alpha_{2}^{j} \leq b<1$, and $\alpha_{1}^{j}, \alpha_{2}^{j}, \alpha_{3}^{j} \in(0,1)$ for all $j=1,2, \ldots$. For all $n \in \mathbb{N}$, let $S_{n}$ and $S$ be $S$-mappings generated by $T_{n}, T_{n-1}, \ldots, T_{1}$ and $\alpha_{n}, \alpha_{n-1}, \ldots, \alpha_{1}$ and $T_{n}, T_{n-1}, \ldots$ and $\alpha_{n}, \alpha_{n-1}, \ldots$, respectively. Then,

(i) $S_{n}$ is nonexpansive and $F\left(S_{n}\right)=\bigcap_{i=1}^{n} F\left(T_{i}\right)$, for all $n \geq 1$,

(ii) for all $x \in C$ and for all positive integer $k$, the $\lim _{n \rightarrow \infty} U_{n, k}$ exists,

(iii) the mapping $S: C \rightarrow C$ defined by

$$
S x:=\lim _{n \rightarrow \infty} S_{n} x=\lim _{n \rightarrow \infty} U_{n, 1} x, \quad \forall x \in C
$$

is a nonexpansive mapping such that $F(S)=\bigcap_{i=1}^{\infty} F\left(T_{i}\right)$, and it is called the S-mapping generated by $T_{n}, T_{n-1}, \ldots$ and $\alpha_{n}, \alpha_{n-1}, \ldots$,

(iv) if $K$ is any bounded subset of $C$, then

$$
\lim _{n \rightarrow \infty} \sup _{x \in K}\left\|S_{n} x-S x\right\|=0
$$

Lemma 2.3 (see [54]). Let $\left\{x_{n}\right\}$ and $\left\{l_{n}\right\}$ be bounded sequences in a Banach space $X$ and let $\left\{\beta_{n}\right\}$ be a sequence in $[0,1]$ with $0<\liminf _{n \rightarrow \infty} \beta_{n} \leq \limsup _{n \rightarrow \infty} \beta_{n}<1$. Suppose that $x_{n+1}=\left(1-\beta_{n}\right) l_{n}+$ $\beta_{n} x_{n}$ for all integers $n \geq 0$ and $\lim \sup _{n \rightarrow \infty}\left(\left\|l_{n+1}-l_{n}\right\|-\left\|x_{n+1}-x_{n}\right\|\right) \leq 0$. Then, $\lim _{n \rightarrow \infty}\left\|l_{n}-x_{n}\right\|=0$.

Lemma 2.4 (see [55]). Let $H$ be a real Hilbert space. Then, the following inequalities hold:

(i) $\|\lambda x+(1-\lambda) y\|^{2}=\lambda\|x\|^{2}+(1-\lambda)\|y\|^{2}-\lambda(1-\lambda)\|x-y\|^{2}, \forall x, y \in H$ and $\lambda \in[0,1]$,

(ii) $\|x+y\|^{2} \leq\|x\|^{2}+2\langle y, x+y\rangle, \forall x, y \in H$.

Lemma 2.5 (see [56]). Let $C$ be a nonempty closed convex subset of a real Hilbert space $H, A: C \rightarrow$ $H$ a mapping, and $W: H \rightarrow 2^{H}$ a maximal monotone mapping. Then,

$$
F\left(J_{r}(I-r A)\right)=(A+W)^{-1}(0), \quad \forall r>0 .
$$

Lemma 2.6 (see [57]). Let $H$ be a real Hilbert space and let $M$ be a maximal monotone operator on $H$. For $r>0$ and $x \in H$, define the resolvent $J_{r} x$. Then, the following holds:

$$
\frac{s-t}{s}\left\langle J_{s} x-J_{t} x, J_{s} x-x\right\rangle \geq\left\|J_{s} x-J_{t} x\right\|^{2}
$$

for all $s, t>0$ and $x \in H$.

For solving the equilibrium problem for bifunction $\Theta: C \times C \rightarrow \mathbb{R}$, let us assume that $\Theta$ satisfies the following conditions: 
(H1) $\Theta(x, x)=0$ for all $x \in C$,

(H2) $\Theta$ is monotone; that is, $\Theta(x, y)+\Theta(y, x) \leq 0$ for all $x, y \in C$,

(H3) for each $y \in C, x \mapsto \Theta(x, y)$ is concave and upper semicontinuous,

(H4) for each $y \in C, x \mapsto \Theta(x, y)$ is convex,

(H5) for each $y \in C, x \mapsto \Theta(x, y)$ is lower semicontinuous.

Definition 2.7. A differentiable function $K: C \rightarrow \mathbb{R}$ on a convex set $C$ is called

(i) convex [2] if

$$
K(y)-K(x) \geq\left\langle K^{\prime}(x), y-x\right\rangle, \quad \forall x, y \in C,
$$

where $K^{\prime}(x)$ is the Fréchet differentiable of $K$ at $x$;

(ii) strongly convex [2] if there exists a constant $\sigma>0$ such that

$$
K(y)-K(x)-\left\langle K^{\prime}(x), x-y\right\rangle \geq\left(\frac{\sigma}{2}\right)\|x-y\|^{2}, \quad \forall x, y \in C
$$

It is easy to see that if $K: C \rightarrow \mathbb{R}$ is a differentiable strongly convex function with constant $\sigma>0$, then $K^{\prime}: C \rightarrow H$ is strongly monotone with constant $\sigma>0$.

Let $\Theta: C \times C \rightarrow \mathbb{R}$ be an equilibrium bifunction satisfying the conditions (H1)-(H5). Let $r$ be any given positive number. For a given point $x \in C$, consider the auxiliary mixed equilibrium problem to finding $y \in C$ such that

$$
\Theta(y, z)+\varphi(z)-\varphi(y)+\frac{1}{r}\left\langle K^{\prime}(y)-K^{\prime}(x), z-y\right\rangle \geq 0, \quad \forall z \in C,
$$

where $K^{\prime}(x)$ is the Fréchet differentiable of $K$ at $x$. Let $V_{r}^{(\Theta, \varphi)}: C \rightarrow C$ be the mapping such that for each $x \in C, V_{r}^{(\Theta, \varphi)}(x)$ is the set of solutions of $\operatorname{MEP}(x, y)$, that is,

$$
V_{r}^{(\Theta, \varphi)}(x)=\left\{y \in C: \Theta(y, z)+\varphi(z)-\varphi(y)+\frac{1}{r}\left\langle K^{\prime}(y)-K^{\prime}(x), z-y\right\rangle \geq 0, \forall z \in C\right\} .
$$

Then, the following conclusion holds.

Lemma 2.8 (see [58]). Let $C$ be a nonempty closed convex subset of a real Hilbert space $H$, and let $\varphi: C \rightarrow \mathbb{R}$ be a lower semicontinuous and convex functional. Let $\Theta: C \times C \rightarrow \mathbb{R}$ be a bifunction satisfying the conditions (H1)-(H5). Assume that

(i) $K: C \rightarrow \mathbb{R}$ is strongly convex with constant $\sigma>0$ and the function $x \mapsto\left\langle y-x, K^{\prime}(x)\right\rangle$ is weakly upper semicontinuous for each $y \in C$;

(ii) for each $x \in C$, there exist a bounded subset $D_{x} \subset C$ and $z_{x}$ such that for all $y \notin D_{x}$,

$$
\Theta\left(y, z_{x}\right)+\varphi\left(z_{x}\right)-\varphi(y)+\frac{1}{r}\left\langle K^{\prime}(y)-K^{\prime}(x), z_{x}-y\right\rangle<0 .
$$


Then, the following holds:

(a) $V_{r}^{(\Theta, \varphi)}$ is single-valued mapping;

(b) $V_{r}^{(\Theta, \varphi)}$ is nonexpansive if $K^{\prime}$ is Lipschitz continuous with constant $v>0$ and

$$
\left\langle K^{\prime}\left(x_{1}\right)-K^{\prime}\left(x_{2}\right), u_{1}-u_{2}\right\rangle \geq\left\langle K^{\prime}\left(u_{1}\right)-K^{\prime}\left(u_{2}\right), u_{1}-u_{2}\right\rangle, \quad \forall x_{1}, x_{2} \in C,
$$

where $u_{i}=V_{r}^{(\Theta, \varphi)}\left(x_{i}\right)$ for $i=1,2$;

(c) $F\left(V_{r}^{(\Theta, \varphi)}\right)=\operatorname{MEP}(\Theta, \varphi)$;

(d) $\operatorname{MEP}(\Theta, \varphi)$ is closed and convex.

In particular, whenever $\Theta: C \times C \rightarrow \mathbb{R}$ is a bifunction satisfying the conditions (H1)(H5) and $K(x)=\|x\|^{2} / 2$, for all $x \in C$, then $V_{r}^{(\Theta, \varphi)}$ is firmly nonexpansive; that is, for any $x, y \in C$,

$$
\left\|V_{r}^{(\Theta, \varphi)} x-V_{r}^{(\Theta, \varphi)} y\right\|^{2} \leq\left\langle V_{r}^{(\Theta, \varphi)} x-V_{r}^{(\Theta, \varphi)} y, x-y\right\rangle .
$$

In this case, $V_{r}^{(\Theta, \varphi)}$ is rewritten as $T_{r}^{(\Theta, \varphi)}$. If, in addition, $\varphi \equiv 0$, then $T_{r}^{(\Theta, \varphi)}$ is rewritten as $T_{r}^{\Theta}$ (see [59, Lemma 2.1] for more details).

Remark 2.9. We remark that Lemma 2.8 is not a consequence of [2, Lemma 3.1] because the condition of the sequential continuity from the weak topology to the strong topology for the derivative $K^{\prime}$ of the function $K: C \rightarrow \mathbb{R}$ does not cover the case $K(x)=\|x\|^{2} / 2$.

Lemma 2.10. Let $C, H, \Theta, \varphi$, and $V_{r}^{(\Theta, \varphi)}$ be as in Lemma 2.8. Then, the following holds:

$$
\begin{aligned}
& \left\langle K^{\prime}\left(V_{s}^{(\Theta, \varphi)} x\right)-K^{\prime}\left(V_{t}^{(\Theta, \varphi)} x\right), V_{s}^{(\Theta, \varphi)} x-V_{t}^{(\Theta, \varphi)} x\right\rangle \\
& \quad \leq \frac{s-t}{s}\left\langle K^{\prime}\left(V_{s}^{(\Theta, \varphi)} x\right)-K^{\prime}(x), V_{s}^{(\Theta, \varphi)} x-V_{t}^{(\Theta, \varphi)} x\right\rangle,
\end{aligned}
$$

for all $s, t>0$ and $x \in C$.

Proof. By similar argument as in the proof of Proposition 1 in [58], for all $s, t>0$ and $x \in C$, let $u=V_{s}^{(\Theta, \varphi)} x$ and $v=V_{t}^{(\Theta, \varphi)} x$; we have

$$
\begin{aligned}
& \Theta(u, y)+\varphi(y)-\varphi(u)+\frac{1}{s}\left\langle K^{\prime}(u)-K^{\prime}(x), y-u\right\rangle \geq 0, \quad \forall x \in C, \\
& \Theta(v, y)+\varphi(y)-\varphi(v)+\frac{1}{t}\left\langle K^{\prime}(v)-K^{\prime}(x), y-v\right\rangle \geq 0, \quad \forall x \in C .
\end{aligned}
$$


Let $y=v$ in (2.14) and $y=u$ in (2.15); we have

$$
\begin{aligned}
& \Theta(u, v)+\varphi(v)-\varphi(u)+\frac{1}{s}\left\langle K^{\prime}(u)-K^{\prime}(x), v-u\right\rangle \geq 0, \\
& \Theta(v, u)+\varphi(u)-\varphi(v)+\frac{1}{t}\left\langle K^{\prime}(v)-K^{\prime}(x), u-v\right\rangle \geq 0 .
\end{aligned}
$$

Adding up the last two inequalities and from the monotonicity of $\Theta$, we obtain that

$$
\frac{1}{s}\left\langle K^{\prime}(u)-K^{\prime}(x), v-u\right\rangle+\frac{1}{t}\left\langle K^{\prime}(v)-K^{\prime}(x), u-v\right\rangle \geq 0 .
$$

It follows that

$$
\left\langle\frac{K^{\prime}(v)-K^{\prime}(x)}{t}-\frac{K^{\prime}(u)-K^{\prime}(x)}{s}, u-v\right\rangle \geq 0
$$

We derive from (2.18) that

$$
\begin{aligned}
0 & \leq\left\langle K^{\prime}(v)-K^{\prime}(x)-\frac{t}{s}\left(K^{\prime}(u)-K^{\prime}(x)\right), u-v\right\rangle \\
& =\left\langle K^{\prime}(v)-K^{\prime}(u)+K^{\prime}(u)-K^{\prime}(x)-\frac{t}{s}\left(K^{\prime}(u)-K^{\prime}(x)\right), u-v\right\rangle \\
& =\left\langle K^{\prime}(v)-K^{\prime}(u)+\left(1-\frac{t}{s}\right)\left(K^{\prime}(u)-K^{\prime}(x)\right), u-v\right\rangle .
\end{aligned}
$$

Hence, we obtain that

$$
\left\langle K^{\prime}(u)-K^{\prime}(v), u-v\right\rangle \leq \frac{s-t}{s}\left\langle K^{\prime}(u)-K^{\prime}(x), u-v\right\rangle .
$$

The following lemma can be found in [60, 61] (see also [62, Lemma 2.2]).

Lemma 2.11. Let $C$ be a nonempty closed convex subset of a real Hilbert space $H$ and $g: C \rightarrow$ $\mathbb{R} \cup\{+\infty\}$ a proper lower semicontinuous differentiable convex function differentiable convex function. If $x^{*}$ is a solution to the minimization problem

$$
g\left(x^{*}\right)=\inf _{x \in C} g(x)
$$

then

$$
\left\langle g^{\prime}(x), x-x^{*}\right\rangle \geq 0, \quad x \in C .
$$


In particular, if $x^{*}$ solves the optimization problem

$$
\min _{x \in \Omega} \frac{\mu}{2}\langle A x, x\rangle+\frac{1}{2}\|x-u\|^{2}-h(x)
$$

then

$$
\left\langle u+(\gamma f-(I+\mu A)) x^{*}, x-x^{*}\right\rangle \leq 0, \quad x \in C,
$$

where $h$ is a potential function for $r f$.

Lemma 2.12 (see [49]). Assume that $\left\{a_{n}\right\}$ is a sequence of nonnegative real numbers such that

$$
a_{n+1} \leq\left(1-\sigma_{n}\right) a_{n}+\delta_{n}
$$

where $\left\{\sigma_{n}\right\}$ is a sequence in $(0,1)$ and $\left\{\delta_{n}\right\}$ is a sequence in $\mathbb{R}$ such that

(i) $\sum_{n=0}^{\infty} \sigma_{n}=\infty$,

(ii) $\lim \sup _{n \rightarrow \infty} \delta_{n} / \sigma_{n} \leq 0$ or $\sum_{n=0}^{\infty}\left|\delta_{n}\right|<\infty$.

Then, $\lim _{n \rightarrow \infty} a_{n}=0$.

\section{Main Results}

Now, we give our main results in this paper.

Theorem 3.1. Let $C$ be a nonempty closed convex subset of a Hilbert space $H$. Let $\varphi_{1}, \varphi_{2}: C \rightarrow \mathbb{R}$ be two lower semicontinuous and convex functionals, and let $\Theta_{1}, \Theta_{2}: C \times C \rightarrow \mathbb{R}$ be two bifunctions satisfying conditions (H1)-(H5). Let $\Psi_{1}, \Psi_{2}, \Phi_{1}, \Phi_{2}: C \rightarrow H$ be $\widetilde{\beta}_{1}$-inverse-strongly monotone mapping, $\tilde{\beta}_{2}$-inverse-strongly monotone mapping, $\tilde{\gamma}_{1}$-inverse-strongly monotone mapping, and $\tilde{\gamma}_{2}$ inverse-strongly monotone mapping, respectively, and let $B_{1}, B_{2}: C \rightarrow H$ be $\tilde{\xi}_{1}$-inverse-strongly monotone mapping and $\tilde{\xi}_{2}$-inverse-strongly monotone mapping, respectively. Let $\left\{T_{i}\right\}_{i=1}^{\infty}: C \rightarrow C$ be an infinite family of nonexpansive mappings, and let $\alpha_{i}=\left(\alpha_{1}^{j}, \alpha_{2}^{j}, \alpha_{3}^{j}\right) \in I \times I \times I$, where $I=[0,1]$, $\alpha_{1}^{j}+\alpha_{2}^{j}+\alpha_{3}^{j}=1, \alpha_{1}^{j}+\alpha_{2}^{j} \leq b<1$, and $\alpha_{1}^{j}, \alpha_{2}^{j}, \alpha_{3}^{j} \in(0,1)$ for all $j=1,2, \ldots$. For all $n \in \mathbb{N}$, let $S_{n}$ and $S$ be $S$-mappings generated by $T_{n}, T_{n-1}, \ldots, T_{1}$ and $\alpha_{n}, \alpha_{n-1}, \ldots, \alpha_{1}$ and $T_{n}, T_{n-1}, \ldots$ and $\alpha_{n}, \alpha_{n-1}, \ldots$, respectively. Let $W_{1}, W_{2}: H \rightarrow 2^{H}$ be two maximal monotone operators such that $D\left(W_{1}\right) \subset C$ and $D\left(W_{2}\right) \subset C$, respectively. Assume that

$$
\begin{aligned}
\Omega:= & \bigcap_{i=1}^{\infty} F\left(T_{i}\right) \cap \operatorname{GMEP}\left(\Theta_{1}, \varphi_{1}, \Psi_{1}+\Phi_{1}\right) \\
& \cap \operatorname{GMEP}\left(\Theta_{2}, \varphi_{2}, \Psi_{2}+\Phi_{2}\right) \cap\left(B_{1}+W_{1}\right)^{-1}(0) \cap\left(B_{2}+W_{2}\right)^{-1}(0) \neq \emptyset .
\end{aligned}
$$


Let $f: C \rightarrow H$ be a contraction mapping with a coefficient $\alpha \in(0,1)$, and let $A: C \rightarrow H$ be a strongly positive linear bounded operator with a coefficient $\bar{\gamma} \in(0,1)$. Let $\mu>0$ and $\gamma>0$ be two constants such that $0<\gamma<(1+\mu) \bar{\gamma} / \alpha$. Let $\left\{x_{n}\right\}$ be a sequence defined by $u, x_{1} \in H$ and

$$
\begin{gathered}
u_{n}=V_{r_{n}}^{\left(\Theta_{2}, \varphi_{2}\right)}\left(x_{n}-r_{n}\left(\Psi_{2}+\Phi_{2}\right) x_{n}\right), \\
v_{n}=V_{\mu_{n}}^{\left(\Theta_{1}, \varphi_{1}\right)}\left(u_{n}-\mu_{n}\left(\Psi_{1}+\Phi_{1}\right) u_{n}\right), \\
y_{n}=J_{t_{n}}\left(v_{n}-t_{n} B_{2} v_{n}\right), \\
x_{n+1}=\epsilon_{n}\left(u+\gamma f\left(S_{n} v_{n}\right)\right)+\beta_{n} x_{n}+\left(\left(1-\beta_{n}\right) I-\epsilon_{n}(I+\mu A)\right) S_{n} J_{s_{n}}\left(y_{n}-s_{n} B_{1} y_{n}\right), \quad \forall n \geq 1,
\end{gathered}
$$

where $J_{s_{n}}=\left(I+s_{n} W_{1}\right)^{-1}, J_{t_{n}}=\left(I+t_{n} W_{2}\right)^{-1},\left\{s_{n}\right\} \subset\left(0,2 \tilde{\xi}_{1}\right),\left\{t_{n}\right\} \subset\left(0,2 \tilde{\xi}_{2}\right),\left\{\mu_{n}\right\} \subset$ $\left(0, \min \left\{\tilde{\beta}_{1}, \tilde{\gamma}_{1}\right\}\right),\left\{r_{n}\right\} \subset\left(0, \min \left\{\tilde{\beta}_{2}, \tilde{\gamma}_{2}\right\}\right),\left\{\epsilon_{n}\right\}$ and $\left\{\beta_{n}\right\} \subset(0,1)$. Assume that the following conditions are satisfied:

(C1) for all $i=1,2, K_{i}: C \rightarrow \mathbb{R}$ is strongly convex with constant $\sigma_{i}>0$ and its derivative $K_{i}^{\prime}$ is Lipschitz continuous with constant $\left.v_{i}\right\rangle 0$ such that the function $x \mapsto\left\langle y-x, K_{i}^{\prime}(x)\right\rangle$ is weakly upper semicontinuous for each $y \in C$,

(C2) for all $i=1,2$ and for each $x \in C$, there exist a bounded subset $D_{x} \subset C$ and $z_{x} \in C$ such that, for all $y \notin D_{x}$,

$$
\Theta_{i}\left(y, z_{x}\right)+\varphi_{i}\left(z_{x}\right)-\varphi_{i}(y)+\frac{1}{r_{i}}\left\langle K_{i}^{\prime}(y)-K_{i}^{\prime}(x), z_{x}-y\right\rangle<0,
$$

(C3) $\lim _{n \rightarrow \infty} \alpha_{n}^{1}=0$,

(C4) $\lim _{n \rightarrow \infty} \epsilon_{n}=0$ and $\sum_{n=1}^{\infty} \epsilon_{n}=\infty$,

(C5) $0<\liminf _{n \rightarrow \infty} \beta_{n} \leq \lim \sup _{n \rightarrow \infty} \beta_{n}<1$,

(C6) $0<\liminf \operatorname{in}_{n \rightarrow \infty} \mu_{n} \leq \lim \sup _{n \rightarrow \infty} \mu_{n}<\min \left\{\tilde{\beta}_{1}, \tilde{\gamma}_{1}\right\}$ and $\lim _{n \rightarrow \infty} \mu_{n} / \mu_{n+1}=1$,

(C7) $0<\liminf _{n \rightarrow \infty} r_{n} \leq \lim \sup _{n \rightarrow \infty} r_{n}<\min \left\{\tilde{\beta}_{2}, \tilde{\gamma}_{2}\right\}$ and $\lim _{n \rightarrow \infty} r_{n} / r_{n+1}=1$,

(C8) $0<\liminf _{n \rightarrow \infty} s_{n} \leq \limsup \sup _{n \rightarrow \infty} s_{n}<2 \tilde{\xi}_{1}$ and $\lim _{n \rightarrow \infty} s_{n} / s_{n+1}=1$,

(C9) $0<\liminf _{n \rightarrow \infty} t_{n} \leq \limsup _{n \rightarrow \infty} t_{n}<2 \tilde{\xi}_{2}$ and $\lim _{n \rightarrow \infty} t_{n} / t_{n+1}=1$.

Then, the sequence $\left\{x_{n}\right\}$ defined by (3.2) converges strongly to $x^{*} \in \Omega$, provided $V_{r, n}^{(\Theta, \varphi)}$ is firmly nonexpansive, where $x^{*}$ solves the following optimization problem:

$$
\min _{x \in \Omega} \frac{\mu}{2}\langle A x, x\rangle+\frac{1}{2}\|x-u\|^{2}-h(x) .
$$

Proof. By the conditions (C4) and (C5), we may assume, without loss of generality, that $\epsilon_{n} \leq$ $\left(1-\beta_{n}\right)(1+\mu\|A\|)^{-1}$ for all $n \in \mathbb{N}$. Since $A$ is a linear bounded self-adjoint operator on $C$, we have

$$
\|A\|=\sup \{|\langle A \tilde{u}, \tilde{u}\rangle|: \tilde{u} \in C,\|\tilde{u}\|=1\} .
$$


Observe that

$$
\begin{aligned}
\left\langle\left(\left(1-\beta_{n}\right) I-\epsilon_{n}(I+\mu A)\right) \tilde{u}, \tilde{u}\right\rangle & =1-\beta_{n}-\epsilon_{n}-\epsilon_{n} \mu\langle A \tilde{u}, \tilde{u}\rangle \\
& \geq 1-\beta_{n}-\epsilon_{n}-\epsilon_{n} \mu\|A\| \\
& \geq 0 .
\end{aligned}
$$

This shows that $\left(1-\beta_{n}\right) I-\epsilon_{n}(I+\mu A)$ is positive. It follows that

$$
\begin{aligned}
\left\|\left(1-\beta_{n}\right) I-\epsilon_{n}(I+\mu A)\right\| & =\sup \left\{\left|\left\langle\left(\left(1-\beta_{n}\right) I-\epsilon_{n}(I+\mu A)\right) \tilde{u}, \tilde{u}\right\rangle\right|: \tilde{u} \in C,\|\tilde{u}\|=1\right\} \\
& =\sup \left\{1-\beta_{n}-\epsilon_{n}-\epsilon_{n} \mu\langle A \tilde{u}, \tilde{u}\rangle: \tilde{u} \in C,\|\tilde{u}\|=1\right\} \\
& \leq 1-\beta_{n}-\epsilon_{n}(1+\mu \bar{\gamma}) \\
& <1-\beta_{n}-\epsilon_{n}(1+\mu) \bar{\gamma} .
\end{aligned}
$$

First, we show that $\left\{x_{n}\right\}$ is bounded. Take $\bar{x} \in \Omega$. Since $0<\mu_{n}<\min \left\{\tilde{\beta}_{1}, \tilde{\gamma}_{1}\right\}$ and by Lemma 2.4 (i), we have

$$
\begin{aligned}
\| v_{n}- & \bar{x} \|^{2} \\
= & \left\|V_{\mu_{n}}^{\left(\Theta_{1}, \varphi_{1}\right)}\left(u_{n}-\mu_{n}\left(\Psi_{1}+\Phi_{1}\right) u_{n}\right)-V_{\mu_{n}}^{\left(\Theta_{1}, \varphi_{1}\right)}\left(\bar{x}-\mu_{n}\left(\Psi_{1}+\Phi_{1}\right) \bar{x}\right)\right\|^{2} \\
\leq & \left\|\left(u_{n}-\mu_{n}\left(\Psi_{1}+\Phi_{1}\right) u_{n}\right)-\left(\bar{x}-\mu_{n}\left(\Psi_{1}+\Phi_{1}\right) \bar{x}\right)\right\|^{2} \\
= & \left\|\left(u_{n}-\bar{x}\right)-\mu_{n}\left[\left(\Psi_{1}+\Phi_{1}\right) u_{n}-\left(\Psi_{1}+\Phi_{1}\right) \bar{x}\right]\right\|^{2} \\
= & \left\|\frac{1}{2}\left(\left(u_{n}-\bar{x}\right)-2 \mu_{n}\left(\Psi_{1} u_{n}-\Psi_{1} \bar{x}\right)\right)+\frac{1}{2}\left(\left(u_{n}-\bar{x}\right)-2 \mu_{n}\left(\Phi_{1} u_{n}-\Phi_{1} \bar{x}\right)\right)\right\|^{2} \\
\leq & \frac{1}{2}\left\|\left(u_{n}-\bar{x}\right)-2 \mu_{n}\left(\Psi_{1} u_{n}-\Psi_{1} \bar{x}\right)\right\|^{2}+\frac{1}{2}\left\|\left(u_{n}-\bar{x}\right)-2 \mu_{n}\left(\Phi_{1} u_{n}-\Phi_{1} \bar{x}\right)\right\|^{2} \\
= & \frac{1}{2}\left(\left\|u_{n}-\bar{x}\right\|^{2}-4 \mu_{n}\left\langle u_{n}-\bar{x}, \Psi_{1} u_{n}-\Psi_{1} \bar{x}\right\rangle+4 \mu_{n}^{2}\left\|\Psi_{1} u_{n}-\Psi_{1} \bar{x}\right\|^{2}\right) \\
& +\frac{1}{2}\left(\left\|u_{n}-\bar{x}\right\|^{2}-4 \mu_{n}\left\langle u_{n}-\bar{x}, \Phi_{1} u_{n}-\Phi_{1} \bar{x}\right\rangle+4 \mu_{n}^{2}\left\|\Phi_{1} u_{n}-\Phi_{1} \bar{x}\right\|^{2}\right) \\
\leq & \frac{1}{2}\left(\left\|u_{n}-\bar{x}\right\|^{2}+4 \mu_{n}\left(\mu_{n}-\tilde{\beta}_{1}\right)\left\|\Psi_{1} u_{n}-\Psi_{1} \bar{x}\right\|^{2}\right) \\
& +\frac{1}{2}\left(\left\|u_{n}-\bar{x}\right\|^{2}+4 \mu_{n}\left(\mu_{n}-\tilde{\gamma}_{1}\right)\left\|\Phi_{1} u_{n}-\Phi_{1} \bar{x}\right\|^{2}\right) \\
= & \left\|u_{n}-\bar{x}\right\|^{2}+2 \mu_{n}\left(\mu_{n}-\tilde{\beta}_{1}\right)\left\|\Psi_{1} u_{n}-\Psi_{1} \bar{x}\right\|^{2}+2 \mu_{n}\left(\mu_{n}-\tilde{\gamma}_{1}\right)\left\|\Phi_{1} u_{n}-\Phi_{1} \bar{x}\right\|^{2} \\
\leq & \left\|u_{n}-\bar{x}\right\|^{2} .
\end{aligned}
$$


In a similar way, we can get

$$
\begin{aligned}
\left\|u_{n}-\bar{x}\right\|^{2} & \left.=\| V_{\left.r_{n}, \Theta_{2}\right)}^{\left(\Theta_{2}\right)}-r_{n}\left(\Psi_{2}+\Phi_{2}\right) x_{n}\right)-V_{r_{n}}^{\left(\Theta_{2}, \varphi_{2}\right)}\left(\bar{x}-r_{n}\left(\Psi_{2}+\Phi_{1}\right) \bar{x}\right) \|^{2} \\
& \leq\left\|x_{n}-\bar{x}\right\|^{2}+2 r_{n}\left(r_{n}-\tilde{\beta}_{2}\right)\left\|\Psi_{2} x_{n}-\Psi_{2} \bar{x}\right\|^{2}+2 r_{n}\left(r_{n}-\tilde{\gamma}_{2}\right)\left\|\Phi_{2} x_{n}-\Phi_{2} \bar{x}\right\|^{2} \\
& \leq\left\|x_{n}-\bar{x}\right\|^{2} .
\end{aligned}
$$

It follows from (3.8), and (3.9) that

$$
\left\|v_{n}-\bar{x}\right\| \leq\left\|u_{n}-\bar{x}\right\| \leq\left\|x_{n}-\bar{x}\right\| .
$$

Setting $z_{n}:=J_{s_{n}}\left(y_{n}-s_{n} B_{1} y_{n}\right)$. Since $0<s_{n}<2 \widetilde{\xi}_{1}$, we have

$$
\begin{aligned}
\left\|z_{n}-\bar{x}\right\|^{2} & =\left\|J_{s_{n}}\left(y_{n}-s_{n} B_{1} y_{n}\right)-J_{s_{n}}\left(\bar{x}-s_{n} B_{1} \bar{x}\right)\right\|^{2} \\
& \leq\left\|\left(y_{n}-s_{n} B_{1} y_{n}\right)-\left(\bar{x}-s_{n} B_{1} \bar{x}\right)\right\|^{2} \\
& =\left\|\left(y_{n}-\bar{x}\right)-s_{n}\left(B_{1} y_{n}-B_{1} \bar{x}\right)\right\|^{2} \\
& =\left\|y_{n}-\bar{x}\right\|^{2}-2 s_{n}\left\langle y_{n}-\bar{x}, B_{1} y_{n}-B_{1} \bar{x}\right\rangle+s_{n}^{2}\left\|B_{1} y_{n}-B_{1} \bar{x}\right\|^{2} \\
& \leq\left\|y_{n}-\bar{x}\right\|^{2}+s_{n}\left(s_{n}-2 \xi_{1}\right)\left\|B_{1} y_{n}-B_{1} \bar{x}\right\|^{2} \\
& \leq\left\|y_{n}-\bar{x}\right\|^{2} .
\end{aligned}
$$

In a similar way, we can get

$$
\begin{aligned}
\left\|y_{n}-\bar{x}\right\|^{2} & =\left\|J_{t_{n}}\left(v_{n}-t_{n} B_{2} v_{n}\right)-J_{t_{n}}\left(\bar{x}-t_{n} B_{2} \bar{x}\right)\right\|^{2} \\
& \leq\left\|v_{n}-\bar{x}\right\|^{2}+t_{n}\left(t_{n}-2 \xi_{2}\right)\left\|B_{2} v_{n}-B_{2} \bar{x}\right\|^{2} \\
& \leq\left\|v_{n}-\bar{x}\right\|^{2} .
\end{aligned}
$$

It follows from (3.10), (3.11) and (3.12) that

$$
\left\|z_{n}-\bar{x}\right\| \leq\left\|y_{n}-\bar{x}\right\| \leq\left\|v_{n}-\bar{x}\right\| \leq\left\|u_{n}-\bar{x}\right\| \leq\left\|x_{n}-\bar{x}\right\| .
$$

Since $x_{n+1}=\epsilon_{n}\left(u+\gamma f\left(S_{n} v_{n}\right)\right)+\beta_{n} x_{n}+\left(\left(1-\beta_{n}\right) I-\epsilon_{n}(I+\mu A)\right) S_{n} z_{n}$, it follows from (3.13) that

$$
\begin{aligned}
\| x_{n+1} & -\bar{x} \| \\
& =\left\|\epsilon_{n} u+\epsilon_{n}\left(\gamma f\left(S_{n} v_{n}\right)-(I+\mu A) \bar{x}\right)+\beta_{n}\left(x_{n}-\bar{x}\right)+\left(\left(1-\beta_{n}\right) I-\epsilon_{n}(I+\mu A)\right)\left(S_{n} z_{n}-\bar{x}\right)\right\| \\
& \leq \epsilon_{n}\|u\|+\epsilon_{n}\left\|\gamma f\left(S_{n} v_{n}\right)-(I+\mu A) \bar{x}\right\|+\beta_{n}\left\|x_{n}-\bar{x}\right\|+\left(1-\beta_{n}-\epsilon_{n}(1+\mu \bar{\gamma})\right)\left\|S_{n} z_{n}-\bar{x}\right\|
\end{aligned}
$$


Abstract and Applied Analysis

$$
\begin{aligned}
\leq & \epsilon_{n}\|u\|+\epsilon_{n} \gamma\left\|f\left(S_{n} v_{n}\right)-f(\bar{x})\right\|+\epsilon_{n}\|\gamma f(\bar{x})-(I+\mu A) \bar{x}\|+\beta_{n}\left\|x_{n}-\bar{x}\right\| \\
& +\left(1-\beta_{n}-\epsilon_{n}(1+\mu) \bar{\gamma}\right)\left\|z_{n}-\bar{x}\right\| \\
\leq & \epsilon_{n}\|u\|+\epsilon_{n} \gamma \alpha\left\|S_{n} v_{n}-\bar{x}\right\|+\epsilon_{n}\|\gamma f(\bar{x})-(I+\mu A) \bar{x}\|+\beta_{n}\left\|x_{n}-\bar{x}\right\| \\
& +\left(1-\beta_{n}-\epsilon_{n}(1+\mu) \bar{\gamma}\right)\left\|x_{n}-\bar{x}\right\| \\
\leq & \left(1-((1+\mu) \bar{\gamma}-\gamma \alpha) \epsilon_{n}\right)\left\|x_{n}-\bar{x}\right\|+\epsilon_{n}\|\gamma f(\bar{x})-(I+\mu A) \bar{x}\|+\epsilon_{n}\|u\| \\
= & \left(1-((1+\mu) \bar{\gamma}-\gamma \alpha) \epsilon_{n}\right)\left\|x_{n}-\bar{x}\right\|+((1+\mu) \bar{\gamma}-\gamma \alpha) \epsilon_{n} \frac{\|\gamma f(\bar{x})-(I+\mu A) \bar{x}\|+\|u\|}{(1+\mu) \bar{\gamma}-\gamma \alpha} .
\end{aligned}
$$

By induction, we have

$$
\left\|x_{n}-\bar{x}\right\| \leq \max \left\{\left\|x_{1}-\bar{x}\right\|, \frac{\|\gamma f(\bar{x})-(I+\mu A) \bar{x}\|+\|u\|}{(1+\mu) \bar{\gamma}-\gamma \alpha}\right\}, \quad \forall n \geq 1 .
$$

Hence, $\left\{x_{n}\right\}$ is bounded, so are $\left\{v_{n}\right\},\left\{y_{n}\right\}$, and $\left\{z_{n}\right\}$.

Next, we show that $\lim _{n \rightarrow \infty}\left\|x_{n+1}-x_{n}\right\|$. Since $u_{n}=V_{r_{n}}^{\left(\Theta_{2}, \varphi_{2}\right)}\left(x_{n}-r_{n}\left(\Psi_{2}+\Phi_{2}\right) x_{n}\right)$ and $u_{n+1}=V_{r_{n+1}}^{\left(\Theta_{2}, \varphi_{2}\right)}\left(x_{n+1}-r_{n+1}\left(\Psi_{2}+\Phi_{2}\right) x_{n+1}\right)$, we have

$$
\begin{aligned}
\| u_{n+1}- & u_{n} \| \\
= & \left\|V_{\left.r_{n+1}, \varphi_{2}\right)}^{\left(\Theta_{2}\right)}\left(x_{n+1}-r_{n+1}\left(\Psi_{2}+\Phi_{2}\right) x_{n+1}\right)-V_{r_{n}}^{\left(\Theta_{2}, \varphi_{2}\right)}\left(x_{n}-r_{n}\left(\Psi_{2}+\Phi_{2}\right) x_{n}\right)\right\| \\
\leq & \left\|V_{r_{n+1}}^{\left(\Theta_{2}, \varphi_{2}\right)}\left(x_{n+1}-r_{n+1}\left(\Psi_{2}+\Phi_{2}\right) x_{n+1}\right)-V_{r_{n+1}}^{\left(\Theta_{2}, \varphi_{2}\right)}\left(x_{n}-r_{n}\left(\Psi_{2}+\Phi_{2}\right) x_{n}\right)\right\| \\
& +\left\|V_{r_{n+1}}^{\left(\Theta_{2}, \varphi_{2}\right)}\left(x_{n}-r_{n}\left(\Psi_{2}+\Phi_{2}\right) x_{n}\right)-V_{r_{n}}^{\left(\Theta_{2}, \varphi_{2}\right)}\left(x_{n}-r_{n}\left(\Psi_{2}+\Phi_{2}\right) x_{n}\right)\right\| \\
\leq & \left\|\left(x_{n+1}-r_{n+1}\left(\Psi_{2}+\Phi_{2}\right) x_{n+1}\right)-\left(x_{n}-r_{n}\left(\Psi_{2}+\Phi_{2}\right) x_{n}\right)\right\| \\
& +\left\|V_{r_{n+1}}^{\left(\Theta_{2}, \varphi_{2}\right)}\left(x_{n}-r_{n}\left(\Psi_{2}+\Phi_{2}\right) x_{n}\right)-V_{r_{n}}^{\left(\Theta_{2}, \varphi_{2}\right)}\left(x_{n}-r_{n}\left(\Psi_{2}+\Phi_{2}\right) x_{n}\right)\right\| \\
= & \left\|x_{n+1}-x_{n}-r_{n+1}\left[\left(\Psi_{2}+\Phi_{2}\right) x_{n+1}-\left(\Psi_{2}+\Phi_{2}\right) x_{n}\right]+\left(r_{n}-r_{n+1}\right)\left(\Psi_{2}+\Phi_{2}\right) x_{n}\right\| \\
& +\left\|V_{r_{n+1}}^{\left(\Theta_{2}, \varphi_{2}\right)}\left(x_{n}-r_{n}\left(\Psi_{2}+\Phi_{2}\right) x_{n}\right)-V_{r_{n}}^{\left(\Theta_{2}, \varphi_{2}\right)}\left(x_{n}-r_{n}\left(\Psi_{2}+\Phi_{2}\right) x_{n}\right)\right\| \\
= & \left\|x_{n+1}-r_{n+1}\left(\Psi_{2}+\Phi_{2}\right) x_{n+1}-\left(x_{n}-r_{n+1}\left(\Psi_{2}+\Phi_{2}\right) x_{n}\right)+\left(r_{n}-r_{n+1}\right)\left(\Psi_{2}+\Phi_{2}\right) x_{n}\right\| \\
& +\left\|V_{r_{n+1}}^{\left(\Theta_{2}, \varphi_{2}\right)}\left(x_{n}-r_{n}\left(\Psi_{2}+\Phi_{2}\right) x_{n}\right)-V_{r_{n}}^{\left(\Theta_{2}, \varphi_{2}\right)}\left(x_{n}-r_{n}\left(\Psi_{2}+\Phi_{2}\right) x_{n}\right)\right\| \\
\leq & \left\|x_{n+1}-x_{n}\right\|+r_{n+1}\left|1-\frac{r_{n}}{r_{n+1}}\right|\left\|\left(\Psi_{2}+\Phi_{2}\right) x_{n}\right\| \\
& +\left\|V_{r_{n+1}}^{\left(\Theta_{2}, \varphi_{2}\right)}\left(x_{n}-r_{n}\left(\Psi_{2}+\Phi_{2}\right) x_{n}\right)-V_{r_{n}}^{\left(\Theta_{2}, \varphi_{2}\right)}\left(x_{n}-r_{n}\left(\Psi_{2}+\Phi_{2}\right) x_{n}\right)\right\| .
\end{aligned}
$$


In a similar way, we can get

$$
\begin{aligned}
\left\|v_{n+1}-v_{n}\right\|= & \left\|V_{\mu_{n+1}}^{\left(\Theta_{1}, \varphi_{1}\right)}\left(u_{n+1}-\mu_{n+1}\left(\Psi_{1}+\Phi_{1}\right) u_{n+1}\right)-V_{\mu_{n}}^{\left(\Theta_{1}, \varphi_{1}\right)}\left(u_{n}-\mu_{n}\left(\Psi_{1}+\Phi_{1}\right) u_{n}\right)\right\| \\
\leq & \left\|u_{n+1}-u_{n}\right\|+\mu_{n+1}\left|1-\frac{\mu_{n}}{\mu_{n+1}}\right|\left\|\left(\Psi_{1}+\Phi_{1}\right) u_{n}\right\| \\
& +\left\|V_{\mu_{n+1}}^{\left(\Theta_{1}, \varphi_{1}\right)}\left(u_{n}-\mu_{n}\left(\Psi_{1}+\Phi_{1}\right) u_{n}\right)-V_{\mu_{n}}^{\left(\Theta_{1}, \varphi_{1}\right)}\left(u_{n}-\mu_{n}\left(\Psi_{1}+\Phi_{1}\right) u_{n}\right)\right\| .
\end{aligned}
$$

Substitution (3.16) into (3.17), we obtain

$$
\begin{aligned}
\left\|v_{n+1}-v_{n}\right\| \leq & \left\|x_{n+1}-x_{n}\right\|+r_{n+1}\left|1-\frac{r_{n}}{r_{n+1}}\right|\left\|\left(\Psi_{2}+\Phi_{2}\right) x_{n}\right\|+\mu_{n+1}\left|1-\frac{\mu_{n}}{\mu_{n+1}}\right|\left\|\left(\Psi_{1}+\Phi_{1}\right) u_{n}\right\| \\
& +\left\|V_{r_{n+1}}^{\left(\Theta_{2}, \varphi_{2}\right)}\left(x_{n}-r_{n}\left(\Psi_{2}+\Phi_{2}\right) x_{n}\right)-V_{r_{n}}^{\left(\Theta_{2}, \varphi_{2}\right)}\left(x_{n}-r_{n}\left(\Psi_{2}+\Phi_{2}\right) x_{n}\right)\right\| \\
& +\left\|V_{\mu_{n+1}}^{\left(\Theta_{1}, \varphi_{1}\right)}\left(u_{n}-\mu_{n}\left(\Psi_{1}+\Phi_{1}\right) u_{n}\right)-V_{\mu_{n}}^{\left(\Theta_{1}, \varphi_{1}\right)}\left(u_{n}-\mu_{n}\left(\Psi_{1}+\Phi_{1}\right) u_{n}\right)\right\| \\
\leq & \left\|x_{n+1}-x_{n}\right\|+\left(\left|1-\frac{r_{n}}{r_{n+1}}\right|+\left|1-\frac{\mu_{n}}{\mu_{n+1}}\right|\right) M_{1} \\
& +\left\|V_{r_{n+1}}^{\left(\Theta_{2}, \varphi_{2}\right)}\left(x_{n}-r_{n}\left(\Psi_{2}+\Phi_{2}\right) x_{n}\right)-V_{r_{n}}^{\left(\Theta_{2}, \varphi_{2}\right)}\left(x_{n}-r_{n}\left(\Psi_{2}+\Phi_{2}\right) x_{n}\right)\right\| \\
& +\left\|V_{\mu_{n+1}}^{\left(\Theta_{1}, \varphi_{1}\right)}\left(u_{n}-\mu_{n}\left(\Psi_{1}+\Phi_{1}\right) u_{n}\right)-V_{\mu_{n}}^{\left(\Theta_{1}, \varphi_{1}\right)}\left(u_{n}-\mu_{n}\left(\Psi_{1}+\Phi_{1}\right) u_{n}\right)\right\|
\end{aligned}
$$

where $M_{1}=\sup _{n \geq 1}\left\{\mu_{n+1}\left\|\left(\Psi_{1}+\Phi_{1}\right) u_{n}\right\|, r_{n+1}\left\|\left(\Psi_{2}+\Phi_{2}\right) x_{n}\right\|\right\}$.

On the other hand, notice from Lemma 2.6 that

$$
\begin{aligned}
\| y_{n+1} & -y_{n} \| \\
= & \left\|J_{t_{n+1}}\left(v_{n+1}-t_{n+1} B_{2} v_{n+1}\right)-J_{t_{n}}\left(v_{n}-t_{n} B_{2} v_{n}\right)\right\| \\
\leq & \left\|J_{t_{n+1}}\left(v_{n+1}-t_{n+1} B_{2} v_{n+1}\right)-J_{t_{n+1}}\left(v_{n}-t_{n} B_{2} v_{n}\right)\right\|+\left\|J_{t_{n+1}}\left(v_{n}-t_{n} B_{2} v_{n}\right)-J_{s_{n}}\left(v_{n}-t_{n} B_{2} v_{n}\right)\right\| \\
\leq & \left\|\left(v_{n+1}-t_{n+1} B_{2} v_{n+1}\right)-\left(v_{n}-t_{n} B_{2} v_{n}\right)\right\|+\left\|J_{t_{n+1}}\left(v_{n}-t_{n} B_{2} v_{n}\right)-J_{s_{n}}\left(v_{n}-t_{n} B_{2} v_{n}\right)\right\| \\
= & \left\|\left(v_{n+1}-t_{n+1} B_{2} v_{n+1}\right)-\left(v_{n}-t_{n+1} B_{2} v_{n}\right)+\left(t_{n}-t_{n+1}\right) B_{2} v_{n}\right\| \\
& +\left\|J_{t_{n+1}}\left(v_{n}-t_{n} B_{2} v_{n}\right)-J_{t_{n}}\left(v_{n}-t_{n} B_{2} v_{n}\right)\right\| \\
\leq & \left\|v_{n+1}-v_{n}\right\|+t_{n+1}\left|1-\frac{t_{n}}{t_{n+1}}\right|\left\|B_{2} v_{n}\right\|+\left|1-\frac{t_{n}}{t_{n+1}}\right|\left\|J_{t_{n+1}}\left(v_{n}-t_{n} B_{2} v_{n}\right)-\left(v_{n}-t_{n} B_{2} v_{n}\right)\right\| \\
\leq & \left\|v_{n+1}-v_{n}\right\|+2\left|1-\frac{t_{n}}{t_{n+1}}\right| M_{2},
\end{aligned}
$$

where $M_{2}>0$ is an appropriate constant such that $M_{2}=\sup _{n \geq 1}\left\{t_{n+1}\left\|B_{2} v_{n}\right\|, \| J_{t_{n+1}}\left(v_{n}-\right.\right.$ $\left.\left.t_{n} B_{2} v_{n}\right)-\left(v_{n}-t_{n} B_{2} v_{n}\right) \|\right\}$. 
In a similar way, we can get from Lemma 2.6 that

$$
\begin{aligned}
\| z_{n+1} & -z_{n} \| \\
& =\left\|J_{s_{n+1}}\left(y_{n+1}-s_{n+1} B_{1} y_{n+1}\right)-J_{s_{n}}\left(y_{n}-s_{n} B_{1} y_{n}\right)\right\| \\
& \leq\left\|y_{n+1}-y_{n}\right\|+s_{n+1}\left|1-\frac{s_{n}}{s_{n+1}}\right|\left\|B_{1} y_{n}\right\|+\left|1-\frac{s_{n}}{s_{n+1}}\right|\left\|J_{s_{n+1}}\left(y_{n}-s_{n} B_{1} y_{n}\right)-\left(y_{n}-s_{n} B_{1} y_{n}\right)\right\| \\
& \leq\left\|y_{n+1}-y_{n}\right\|+2\left|1-\frac{s_{n}}{s_{n+1}}\right| M_{3},
\end{aligned}
$$

where $M_{3}>0$ is an appropriate constant such that $M_{3}=\sup _{n \geq 1}\left\{s_{n+1}\left\|B_{1} y_{n}\right\|, \| J_{s_{n+1}}\left(y_{n}-\right.\right.$ $\left.\left.s_{n} B_{1} y_{n}\right)-\left(y_{n}-s_{n} B_{1} y_{n}\right) \|\right\}$. It follows from (3.18) and (3.19) that

$$
\begin{aligned}
\left\|z_{n+1}-z_{n}\right\| \leq & \left\|x_{n+1}-x_{n}\right\|+\left(\left|1-\frac{r_{n}}{r_{n+1}}\right|+\left|1-\frac{\mu_{n}}{\mu_{n+1}}\right|+2\left|1-\frac{t_{n}}{t_{n+1}}\right|+2\left|1-\frac{s_{n}}{s_{n+1}}\right|\right) L \\
& +\left\|V_{r_{n+1}}^{\left(\Theta_{2}, \varphi_{2}\right)}\left(x_{n}-r_{n}\left(\Psi_{2}+\Phi_{2}\right) x_{n}\right)-V_{r_{n}}^{\left(\Theta_{2}, \varphi_{2}\right)}\left(x_{n}-r_{n}\left(\Psi_{2}+\Phi_{2}\right) x_{n}\right)\right\| \\
& +\left\|V_{\mu_{n+1}}^{\left(\Theta_{1}, \varphi_{1}\right)}\left(u_{n}-\mu_{n}\left(\Psi_{1}+\Phi_{1}\right) u_{n}\right)-V_{\mu_{n}}^{\left(\Theta_{1}, \varphi_{1}\right)}\left(u_{n}-\mu_{n}\left(\Psi_{1}+\Phi_{1}\right) u_{n}\right)\right\|,
\end{aligned}
$$

where $L=\max \left\{M_{1}, M_{2}, M_{3}\right\}$.

Define the sequence $\left\{l_{n}\right\}$ by $l_{n}:=\left(x_{n+1}-\beta_{n} x_{n}\right) /\left(1-\beta_{n}\right)$, for all $n \in \mathbb{N}$. Then, $x_{n+1}=$ $\beta_{n} x_{n}+\left(1-\beta_{n}\right) l_{n}$, for all $n \in \mathbb{N}$, we note that

$$
\begin{aligned}
l_{n+1}-l_{n}= & \frac{x_{n+2}-\beta_{n+1} x_{n+1}}{1-\beta_{n+1}}-\frac{x_{n+1}-\beta_{n} x_{n}}{1-\beta_{n}} \\
= & \frac{\epsilon_{n+1}\left(u+\gamma f\left(S_{n+1} v_{n+1}\right)\right)+\left(\left(1-\beta_{n+1}\right) I-\epsilon_{n+1}(I+\mu A)\right) S_{n+1} z_{n+1}}{1-\beta_{n+1}} \\
& -\frac{\epsilon_{n}\left(u+\gamma f\left(S_{n} v_{n}\right)\right)+\left(\left(1-\beta_{n}\right) I-\epsilon_{n}(I+\mu A)\right) S_{n} z_{n}}{1-\beta_{n}} \\
= & \frac{\epsilon_{n+1}}{1-\beta_{n+1}}\left(u+\gamma f\left(S_{n+1} v_{n+1}\right)\right)-\frac{\epsilon_{n}}{1-\beta_{n}}\left(u+\gamma f\left(S_{n} v_{n}\right)\right)+\frac{\epsilon_{n}}{1-\beta_{n}}(I+\mu A) S_{n} z_{n} \\
& -\frac{\epsilon_{n+1}}{1-\beta_{n+1}}(I+\mu A) S_{n+1} z_{n+1}+S_{n+1} z_{n+1}-S_{n} z_{n} .
\end{aligned}
$$

It follows that

$$
\begin{aligned}
\left\|l_{n+1}-l_{n}\right\| \leq & \frac{\epsilon_{n+1}}{1-\beta_{n+1}}\left\|u+\gamma f\left(S_{n+1} v_{n+1}\right)-(I-\mu A) S_{n+1} z_{n+1}\right\| \\
& +\frac{\epsilon_{n}}{1-\beta_{n}}\left\|(I+\mu A) S_{n} z_{n}-u-\gamma f\left(S_{n} v_{n}\right)\right\|+\left\|S_{n+1} z_{n+1}-S_{n} z_{n}\right\| .
\end{aligned}
$$


Now, we estimate $\left\|S_{n+1} z_{n}-S_{n} z_{n}\right\|$. By definition of $S_{n}$, for all $n \in \mathbb{N}$ and for all $\omega \in \Omega$, we have

$$
\begin{aligned}
\left\|S_{n+1} z_{n}-S_{n} z_{n}\right\| & =\left\|U_{n+1,1} z_{n}-U_{n, 1} z_{n}\right\| \\
& =\left\|\alpha_{1}^{1} T_{1} U_{n+1,2} z_{n}+\alpha_{2}^{1} U_{n+1,2} z_{n}+\alpha_{3}^{1} z_{n}-\alpha_{1}^{1} T_{1} U_{n, 2} z_{n}-\alpha_{2}^{1} U_{n, 2} z_{n}-\alpha_{3}^{1} z_{n}\right\| \\
& =\left(\alpha_{1}^{1}+\alpha_{2}^{1}\right)\left\|T_{1} U_{n+1,2} z_{n}-T_{1} U_{n, 2} z_{n}\right\| \\
& \leq\left(1-\alpha_{3}^{1}\right)\left\|U_{n+1,2} z_{n}-U_{n, 2} z_{n}\right\| \\
& \leq\left(1-\alpha_{3}^{1}\right)\left(1-\alpha_{3}^{2}\right)\left\|U_{n+1,3} z_{n}-U_{n, 3} z_{n}\right\| \\
& =\prod_{i=1}^{2}\left(1-\alpha_{3}^{i}\right)\left\|U_{n+1,3} z_{n}-U_{n, 3} z_{n}\right\| \\
& \vdots \\
& \leq \prod_{i=1}^{n}\left(1-\alpha_{3}^{i}\right)\left\|U_{n+1, n+1} z_{n}-U_{n, n+1} z_{n}\right\| \\
& \leq\left\|U_{n+1, n+1} z_{n}-z_{n}\right\| \\
& =\left\|\alpha_{1}^{n+1} T_{n+1} z_{n}+\left(1-\alpha_{1}^{n+1}\right) z_{n}-z_{n}\right\| \\
& =\alpha_{1}^{n+1}\left\|T_{n+1} z_{n}-z_{n}\right\| \\
& \leq \alpha_{1}^{n+1}\left(\left\|T_{n+1} z_{n}-T_{n+1} \omega\right\|+\left\|T_{n+1} \omega-z_{n}\right\|\right) \\
& \leq 2 \alpha_{1}^{n+1}\left\|z_{n}-\omega\right\| .
\end{aligned}
$$

By the condition (C3), we obtain that

$$
\lim _{n \rightarrow \infty}\left\|S_{n+1} z_{n}-S_{n} z_{n}\right\|=0
$$

It follows from (3.21) that

$$
\begin{aligned}
\left\|S_{n+1} z_{n+1}-S_{n} z_{n}\right\| \leq & \left\|S_{n+1} z_{n+1}-S_{n+1} z_{n}\right\|+\left\|S_{n+1} z_{n}-S_{n} z_{n}\right\| \\
\leq & \left\|z_{n+1}-z_{n}\right\|+\left\|S_{n+1} z_{n}-S_{n} z_{n}\right\| \\
\leq & \left\|x_{n+1}-x_{n}\right\|+\left(\left|1-\frac{r_{n}}{r_{n+1}}\right|+\left|1-\frac{\mu_{n}}{\mu_{n+1}}\right|+2\left|1-\frac{t_{n}}{t_{n+1}}\right|+2\left|1-\frac{S_{n}}{S_{n+1}}\right|\right) L \\
& +\left\|V_{r_{n+1}}^{\left(\Theta_{2}, \varphi_{2}\right)}\left(x_{n}-r_{n}\left(\Psi_{2}+\Phi_{2}\right) x_{n}\right)-V_{r_{n}}^{\left(\Theta_{2}, \varphi_{2}\right)}\left(x_{n}-r_{n}\left(\Psi_{2}+\Phi_{2}\right) x_{n}\right)\right\| \\
& +\left\|V_{\mu_{n+1}}^{\left(\Theta_{1}, \varphi_{1}\right)}\left(u_{n}-\mu_{n}\left(\Psi_{1}+\Phi_{1}\right) u_{n}\right)-V_{\mu_{n}}^{\left(\Theta_{1}, \varphi_{1}\right)}\left(u_{n}-\mu_{n}\left(\Psi_{1}+\Phi_{1}\right) u_{n}\right)\right\| .
\end{aligned}
$$


It follows from (3.23) and (3.26) that

$$
\begin{aligned}
\| l_{n+1}- & l_{n}\|-\| x_{n+1}-x_{n} \| \\
\leq & \frac{\epsilon_{n+1}}{1-\beta_{n+1}}\left\|u+\gamma f\left(S_{n+1} v_{n+1}\right)-(I-\mu A) S_{n+1} z_{n+1}\right\| \\
& +\frac{\epsilon_{n}}{1-\beta_{n}}\left\|(I+\mu A) S_{n} z_{n}-u-\gamma f\left(S_{n} v_{n}\right)\right\|+\left\|S_{n+1} z_{n+1}-S_{n} z_{n}\right\|-\left\|x_{n+1}-x_{n}\right\| \\
\leq & \frac{\epsilon_{n+1}}{1-\beta_{n+1}}\left\|u+\gamma f\left(S_{n+1} v_{n+1}\right)-(I-\mu A) S_{n+1} z_{n+1}\right\| \\
& +\frac{\epsilon_{n}}{1-\beta_{n}}\left\|(I+\mu A) S_{n} z_{n}-u-\gamma f\left(S_{n} v_{n}\right)\right\| \\
& +\left(\left|1-\frac{r_{n}}{r_{n+1}}\right|+\left|1-\frac{\mu_{n}}{\mu_{n+1}}\right|+2\left|1-\frac{t_{n}}{t_{n+1}}\right|+2\left|1-\frac{S_{n}}{S_{n+1}}\right|\right) L \\
& +\left\|V_{r_{n+1}}^{\left(\Theta_{2}, \varphi_{2}\right)}\left(x_{n}-r_{n}\left(\Psi_{2}+\Phi_{2}\right) x_{n}\right)-V_{r_{n}}^{\left(\Theta_{2}, \varphi_{2}\right)}\left(x_{n}-r_{n}\left(\Psi_{2}+\Phi_{2}\right) x_{n}\right)\right\| \\
& +\left\|V_{\mu_{n+1}}^{\left(\Theta_{1}, \varphi_{1}\right)}\left(u_{n}-\mu_{n}\left(\Psi_{1}+\Phi_{1}\right) u_{n}\right)-V_{\mu_{n}}^{\left(\Theta_{1}, \varphi_{1}\right)}\left(u_{n}-\mu_{n}\left(\Psi_{1}+\Phi_{1}\right) u_{n}\right)\right\| \\
& +\left\|S_{n+1} z_{n}-S_{n} z_{n}\right\| .
\end{aligned}
$$

Note that $0<\liminf _{n \rightarrow \infty} r_{n} \leq \lim \sup _{n \rightarrow \infty} r_{n}<\min \left\{\tilde{\beta}_{2}, \tilde{\gamma}_{2}\right\}$ and $\lim _{n \rightarrow \infty} r_{n} / r_{n+1}=1$. Since $K_{1}^{\prime}$ is $\sigma_{1}$-strongly monotone and $v_{1}$-Lipschitz continuous, then, from Lemma 2.10, we obtain that

$$
\lim _{n \rightarrow \infty}\left\|V_{r_{n+1}}^{\left(\Theta_{2}, \varphi_{2}\right)}\left(x_{n}-r_{n}\left(\Psi_{2}+\Phi_{2}\right) x_{n}\right)-V_{r_{n}}^{\left(\Theta_{2}, \varphi_{2}\right)}\left(x_{n}-r_{n}\left(\Psi_{2}+\Phi_{2}\right) x_{n}\right)\right\|=0 .
$$

In a similar way, we can get

$$
\lim _{n \rightarrow \infty}\left\|V_{\mu_{n+1}}^{\left(\Theta_{1}, \varphi_{1}\right)}\left(u_{n}-\mu_{n}\left(\Psi_{1}+\Phi_{1}\right) u_{n}\right)-V_{\mu_{n}}^{\left(\Theta_{1}, \varphi_{1}\right)}\left(u_{n}-\mu_{n}\left(\Psi_{1}+\Phi_{1}\right) u_{n}\right)\right\|=0
$$

Consequently, it follows from (3.25), (3.27), (3.28), (3.29), and the conditions (C4)-(C9) that

$$
\limsup _{n \rightarrow \infty}\left(\left\|l_{n+1}-l_{n}\right\|-\left\|x_{n+1}-x_{n}\right\|\right) \leq 0
$$

Hence, by Lemma 2.3, we have

$$
\lim _{n \rightarrow \infty}\left\|l_{n}-x_{n}\right\|=0
$$

Moreover, we have

$$
\lim _{n \rightarrow \infty}\left\|x_{n+1}-x_{n}\right\|=\lim _{n \rightarrow \infty}\left(1-\beta_{n}\right)\left\|l_{n}-x_{n}\right\|=0 .
$$


Since

$$
x_{n+1}-x_{n}=\epsilon_{n}\left(u+\gamma f\left(S_{n} v_{n}\right)-(I+\mu A) x_{n}\right)+\left(\left(1-\beta_{n}\right) I-\epsilon_{n}(I+\mu A)\right)\left(S_{n} z_{n}-x_{n}\right)
$$

it follows that

$$
\left(1-\beta_{n}-\epsilon_{n}(1+\mu) \bar{\gamma}\right)\left\|x_{n}-S_{n} z_{n}\right\| \leq\left\|x_{n+1}-x_{n}\right\|+\epsilon_{n}\left\|u+\gamma f\left(S_{n} v_{n}\right)-(I+\mu A) x_{n}\right\| .
$$

It follows from (3.32) and the conditions (C4) and (C5) that

$$
\lim _{n \rightarrow \infty}\left\|x_{n}-S_{n} z_{n}\right\|=0
$$

Next, we show that

$$
\begin{aligned}
& \lim _{n \rightarrow \infty}\left\|\Psi_{1} u_{n}-\Psi_{1} \bar{x}\right\|=\lim _{n \rightarrow \infty}\left\|\Phi_{1} u_{n}-\Phi_{1} \bar{x}\right\|=0, \\
& \lim _{n \rightarrow \infty}\left\|\Psi_{2} x_{n}-\Psi_{2} \bar{x}\right\|=\lim _{n \rightarrow \infty}\left\|\Phi_{2} x_{n}-\Phi_{2} \bar{x}\right\|=0, \\
& \lim _{n \rightarrow \infty}\left\|B_{1} y_{n}-B_{1} \bar{x}\right\|=\lim _{n \rightarrow \infty}\left\|B_{2} v_{n}-B_{1} \bar{x}\right\|=0 .
\end{aligned}
$$

From (3.8), (3.9), (3.11), and (3.12), we have

$$
\begin{aligned}
\left\|z_{n}-\bar{x}\right\|^{2} \leq & \left\|y_{n}-\bar{x}\right\|^{2}+s_{n}\left(s_{n}-2 \tilde{\xi}_{1}\right)\left\|B_{1} y_{n}-B_{1} \bar{x}\right\|^{2} \\
\leq & \left\|v_{n}-\bar{x}\right\|^{2}+t_{n}\left(t_{n}-2 \tilde{\xi}_{2}\right)\left\|B_{2} v_{n}-B_{2} \bar{x}\right\|^{2}+s_{n}\left(s_{n}-2 \tilde{\xi}_{1}\right)\left\|B_{1} y_{n}-B_{1} \bar{x}\right\|^{2} \\
\leq & \left\|u_{n}-\bar{x}\right\|^{2}+2 \mu_{n}\left(\mu_{n}-\tilde{\beta}_{1}\right)\left\|\Psi_{1} u_{n}-\Psi_{1} \bar{x}\right\|^{2}+2 \mu_{n}\left(\mu_{n}-\tilde{\gamma}_{1}\right)\left\|\Phi_{1} u_{n}-\Phi_{1} \bar{x}\right\|^{2} \\
& +t_{n}\left(t_{n}-2 \tilde{\xi}_{2}\right)\left\|B_{2} v_{n}-B_{2} \bar{x}\right\|^{2}+s_{n}\left(s_{n}-2 \tilde{\xi}_{1}\right)\left\|B_{1} y_{n}-B_{1} \bar{x}\right\|^{2} \\
\leq & \left\|x_{n}-\bar{x}\right\|^{2}+2 r_{n}\left(r_{n}-\tilde{\beta}_{2}\right)\left\|\Psi_{2} x_{n}-\Psi_{2} \bar{x}\right\|^{2}+2 r_{n}\left(r_{n}-\tilde{\gamma}_{2}\right)\left\|\Phi_{2} x_{n}-\Phi_{2} \bar{x}\right\|^{2} \\
& +2 \mu_{n}\left(\mu_{n}-\tilde{\beta}_{1}\right)\left\|\Psi_{1} u_{n}-\Psi_{1} \bar{x}\right\|^{2}+2 \mu_{n}\left(\mu_{n}-\tilde{\gamma}_{1}\right)\left\|\Phi_{1} u_{n}-\Phi_{1} \bar{x}\right\|^{2} \\
& +t_{n}\left(t_{n}-2 \tilde{\xi}_{2}\right)\left\|B_{2} v_{n}-B_{2} \bar{x}\right\|^{2}+s_{n}\left(s_{n}-2 \tilde{\xi}_{1}\right)\left\|B_{1} y_{n}-B_{1} \bar{x}\right\|^{2} .
\end{aligned}
$$


On the other hand, from Lemma 2.4 (ii), we have

$$
\begin{aligned}
\| x_{n+1}- & \bar{x} \|^{2} \\
= & \left\|\epsilon_{n}\left(u+\gamma f\left(S_{n} v_{n}\right)-(I+\mu A) \bar{x}\right)+\beta_{n}\left(x_{n}-S_{n} z_{n}\right)+\left(I-\epsilon_{n}(I+\mu A)\right)\left(S_{n} z_{n}-\bar{x}\right)\right\|^{2} \\
\leq & \left\|\left(I+\epsilon_{n}(I+\mu A)\right)\left(S_{n} z_{n}-\bar{x}\right)+\beta_{n}\left(x_{n}-S_{n} z_{n}\right)\right\|^{2} \\
& +2 \epsilon_{n}\left\langle u+\gamma f\left(S_{n} v_{n}\right)-(I+\mu A) \bar{x}, x_{n+1}-\bar{x}\right\rangle \\
\leq & {\left[\left\|\left(I-\epsilon_{n}(I+\mu A)\right)\left(S_{n} z_{n}-\bar{x}\right)\right\|+\beta_{n}\left\|x_{n}-S_{n} z_{n}\right\|\right]^{2} } \\
& +2 \epsilon_{n}\left\langle u+\gamma f\left(S_{n} v_{n}\right)-(I+\mu A) \bar{x}, x_{n+1}-\bar{x}\right\rangle \\
\leq & {\left[\left(1-\epsilon_{n}-\epsilon_{n} \mu \bar{\gamma}\right)\left\|z_{n}-\bar{x}\right\|+\beta_{n}\left\|x_{n}-S_{n} z_{n}\right\|\right]^{2} } \\
& +2 \epsilon_{n}\left\|u+\gamma f\left(S_{n} v_{n}\right)-(I+\mu A) \bar{x}\right\|\left\|x_{n+1}-\bar{x}\right\| \\
\leq & \left(1-\epsilon_{n}-\epsilon_{n} \mu \bar{\gamma}\right)^{2}\left\|z_{n}-\bar{x}\right\|^{2}+c_{n}
\end{aligned}
$$

where

$$
\begin{aligned}
c_{n}:= & \beta_{n}^{2}\left\|x_{n}-S_{n} z_{n}\right\|^{2}+2\left(1-\epsilon_{n}-\epsilon_{n} \mu \bar{\gamma}\right) \beta_{n}\left\|z_{n}-\bar{x}\right\|\left\|x_{n}-S_{n} z_{n}\right\| \\
& +2 \epsilon_{n}\left\|u+\gamma f\left(S_{n} v_{n}\right)-(I+\mu A) \bar{x}\right\|\left\|x_{n+1}-\bar{x}\right\| .
\end{aligned}
$$

It follows from the condition (C4) and (3.35) that

$$
\lim _{n \rightarrow \infty} c_{n}=0 .
$$

Substituting (3.37) into (3.38), we have

$$
\begin{aligned}
\left\|x_{n+1}-\bar{x}\right\|^{2} \leq & \left(1-\epsilon_{n}-\epsilon_{n} \mu \bar{\gamma}\right)^{2} \\
\times\{\| & \left\|x_{n}-\bar{x}\right\|^{2}+2 r_{n}\left(r_{n}-\tilde{\beta}_{2}\right)\left\|\Psi_{2} x_{n}-\Psi_{2} \bar{x}\right\|^{2}+2 r_{n}\left(r_{n}-\tilde{\gamma}_{2}\right)\left\|\Phi_{2} x_{n}-\Phi_{2} \bar{x}\right\|^{2} \\
& +2 \mu_{n}\left(\mu_{n}-\tilde{\beta}_{1}\right)\left\|\Psi_{1} u_{n}-\Psi_{1} \bar{x}\right\|^{2}+2 \mu_{n}\left(\mu_{n}-\tilde{\gamma}_{1}\right)\left\|\Phi_{1} u_{n}-\Phi_{1} \bar{x}\right\|^{2} \\
& \left.+t_{n}\left(t_{n}-2 \tilde{\xi}_{2}\right)\left\|B_{2} v_{n}-B_{2} \bar{x}\right\|^{2}+s_{n}\left(s_{n}-2 \tilde{\xi}_{1}\right)\left\|B_{1} y_{n}-B_{1} \bar{x}\right\|^{2}\right\}+c_{n}
\end{aligned}
$$


which in turn implies that

$$
\begin{gathered}
\left(1-\epsilon_{n}-\epsilon_{n} \mu \bar{\gamma}\right)^{2}\left\{2 r_{n}\left(\tilde{\beta}_{2}-r_{n}\right)\left\|\Psi_{2} x_{n}-\Psi_{2} \bar{x}\right\|^{2}+2 r_{n}\left(\tilde{\gamma}_{2}-r_{n}\right)\left\|\Phi_{2} x_{n}-\Phi_{2} \bar{x}\right\|^{2}\right. \\
+2 \mu_{n}\left(\tilde{\beta}_{1}-\mu_{n}\right)\left\|\Psi_{1} u_{n}-\Psi_{1} \bar{x}\right\|^{2}+2 \mu_{n}\left(\tilde{\gamma}_{1}-\mu_{n}\right)\left\|\Phi_{1} u_{n}-\Phi_{1} \bar{x}\right\|^{2} \\
\left.+t_{n}\left(2 \tilde{\xi}_{2}-t_{n}\right)\left\|B_{2} v_{n}-B_{2} \bar{x}\right\|^{2}+s_{n}\left(2 \tilde{\xi}_{1}-s_{n}\right)\left\|B_{1} y_{n}-B_{1} \bar{x}\right\|^{2}\right\} \\
\leq\left(1-\epsilon_{n}-\epsilon_{n} \mu \bar{\gamma}\right)^{2}\left\|x_{n}-\bar{x}\right\|^{2}-\left\|x_{n+1}-\bar{x}\right\|^{2}+c_{n} \\
=\left\{1-2 \epsilon_{n}(1+\mu \bar{\gamma})+\epsilon_{n}^{2}(1+\mu \bar{\gamma})^{2}\right\}\left\|x_{n}-\bar{x}\right\|^{2}-\left\|x_{n+1}-\bar{x}\right\|^{2}+c_{n} \\
\leq\left\|x_{n}-\bar{x}\right\|^{2}-\left\|x_{n+1}-\bar{x}\right\|^{2}+\epsilon_{n}^{2}(1+\mu \bar{\gamma})^{2}\left\|x_{n}-\bar{x}\right\|^{2}+c_{n} \\
\leq\left(\left\|x_{n}-\bar{x}\right\|+\left\|x_{n+1}-\bar{x}\right\|\right)\left\|x_{n+1}-x_{n}\right\|+\epsilon_{n}^{2}(1+\mu \bar{\gamma})^{2}\left\|x_{n}-\bar{x}\right\|^{2}+c_{n} .
\end{gathered}
$$

It follows from the conditions (C4)-(C9), (3.32), and (3.40) that

$$
\begin{aligned}
& \lim _{n \rightarrow \infty}\left\|\Psi_{1} u_{n}-\Psi_{1} \bar{x}\right\|=\lim _{n \rightarrow \infty}\left\|\Phi_{1} u_{n}-\Phi_{1} \bar{x}\right\|=0, \\
& \lim _{n \rightarrow \infty}\left\|\Psi_{2} x_{n}-\Psi_{2} \bar{x}\right\|=\lim _{n \rightarrow \infty}\left\|\Phi_{2} x_{n}-\Phi_{2} \bar{x}\right\|=0, \\
& \lim _{n \rightarrow \infty}\left\|B_{1} y_{n}-B_{1} \bar{x}\right\|=\lim _{n \rightarrow \infty}\left\|B_{2} v_{n}-B_{1} \bar{x}\right\|=0 .
\end{aligned}
$$

Next, we show that $\lim _{n \rightarrow \infty}\left\|S z_{n}-z_{n}\right\|=0$. Since $J_{s_{n}}$ is firmly nonexpansive, we have

$$
\begin{aligned}
\left\|z_{n}-\bar{x}\right\|^{2}= & \left\|J_{s_{n}}\left(y_{n}-s_{n} B_{1} y_{n}\right)-J_{s_{n}}\left(\bar{x}-s_{n} B_{1} \bar{x}\right)\right\|^{2} \\
\leq & \left\langle\left(y_{n}-s_{n} B_{1} y_{n}\right)-\left(\bar{x}-s_{n} B_{1} \bar{x}\right), z_{n}-\bar{x}\right\rangle \\
= & \frac{1}{2}\left(\left\|\left(y_{n}-s_{n} B_{1} y_{n}\right)-\left(\bar{x}-s_{n} B_{1} \bar{x}\right)\right\|^{2}+\left\|z_{n}-\bar{x}\right\|^{2}\right. \\
& \left.\quad-\left\|\left(y_{n}-s_{n} B_{1} y_{n}\right)-\left(\bar{x}-s_{n} B_{1} \bar{x}\right)-\left(z_{n}-\bar{x}\right)\right\|^{2}\right) \\
\leq & \frac{1}{2}\left(\left\|y_{n}-\bar{x}\right\|^{2}+\left\|z_{n}-\bar{x}\right\|^{2}-\left\|y_{n}-z_{n}-s_{n}\left(B_{1} y_{n}-B_{1} \bar{x}\right)\right\|^{2}\right) \\
= & \frac{1}{2}\left(\left\|y_{n}-\bar{x}\right\|^{2}+\left\|z_{n}-\bar{x}\right\|^{2}-\left\|y_{n}-z_{n}\right\|^{2}\right. \\
& \left.+2 s_{n}\left\langle y_{n}-z_{n}, B_{1} y_{n}-B_{1} \bar{x}\right\rangle-s_{n}^{2}\left\|B_{1} y_{n}-B_{1} \bar{x}\right\|^{2}\right),
\end{aligned}
$$

which in turn implies that

$$
\begin{aligned}
\left\|z_{n}-\bar{x}\right\|^{2} & \leq\left\|y_{n}-\bar{x}\right\|^{2}-\left\|y_{n}-z_{n}\right\|^{2}+2 s_{n}\left\langle y_{n}-z_{n}, B_{1} y_{n}-B_{1} \bar{x}\right\rangle-s_{n}^{2}\left\|B_{1} y_{n}-B_{1} \bar{x}\right\|^{2} \\
& \leq\left\|x_{n}-\bar{x}\right\|^{2}-\left\|y_{n}-z_{n}\right\|^{2}+2 s_{n}\left\|y_{n}-z_{n}\right\|\left\|B_{1} y_{n}-B_{1} \bar{x}\right\| .
\end{aligned}
$$


Substituting (3.45) into (3.38), we have

$$
\left\|x_{n+1}-\bar{x}\right\|^{2} \leq\left(1-\epsilon_{n}-\epsilon_{n} \mu \bar{\gamma}\right)^{2}\left\{\left\|x_{n}-\bar{x}\right\|^{2}-\left\|y_{n}-z_{n}\right\|^{2}+2 s_{n}\left\|y_{n}-z_{n}\right\|\left\|B_{1} y_{n}-B_{1} \bar{x}\right\|\right\}+c_{n}
$$

which in turn implies that

$$
\begin{aligned}
\left(1-\epsilon_{n}-\epsilon_{n} \mu \bar{\gamma}\right)^{2}\left\|y_{n}-z_{n}\right\|^{2} \leq & \left(1-\epsilon_{n}-\epsilon_{n} \mu \bar{\gamma}\right)^{2}\left\|x_{n}-\bar{x}\right\|^{2}-\left\|x_{n+1}-\bar{x}\right\|^{2} \\
& +2\left(1-\epsilon_{n}-\epsilon_{n} \mu \bar{\gamma}\right)^{2} s_{n}\left\|y_{n}-z_{n}\right\|\left\|B_{1} y_{n}-B_{1} \bar{x}\right\|+c_{n} \\
\leq & \left\|x_{n}-\bar{x}\right\|^{2}-\left\|x_{n+1}-\bar{x}\right\|^{2}+\epsilon_{n}^{2}(1+\mu \bar{\gamma})^{2}\left\|x_{n}-\bar{x}\right\|^{2} \\
& +2 s_{n}\left(1-\epsilon_{n}-\epsilon_{n} \mu \bar{\gamma}\right)^{2}\left\|y_{n}-z_{n}\right\|\left\|B_{1} y_{n}-B_{1} \bar{x}\right\|+c_{n} \\
\leq & \left(\left\|x_{n}-\bar{x}\right\|+\left\|x_{n+1}-\bar{x}\right\|\right)\left\|x_{n+1}-x_{n}\right\| \\
& +\epsilon_{n}^{2}(1+\mu \bar{\gamma})^{2}\left\|x_{n}-\bar{x}\right\|^{2} \\
& +2 s_{n}\left(1-\epsilon_{n}-\epsilon_{n} \mu \bar{\gamma}\right)^{2}\left\|y_{n}-z_{n}\right\|\left\|B_{1} y_{n}-B_{1} \bar{x}\right\|+c_{n} .
\end{aligned}
$$

Since $\epsilon_{n} \rightarrow 0,\left\|B_{1} y_{n}-B_{1} \bar{x}\right\| \rightarrow 0$ and from (3.32), we obtain that

$$
\lim _{n \rightarrow \infty}\left\|y_{n}-z_{n}\right\|=0
$$

In a similar way, we can get

$$
\left\|y_{n}-\bar{x}\right\|^{2} \leq\left\|x_{n}-\bar{x}\right\|^{2}-\left\|v_{n}-y_{n}\right\|^{2}+2 t_{n}\left\|v_{n}-y_{n}\right\|\left\|B_{2} v_{n}-B_{2} \bar{x}\right\| .
$$

It follows from (3.38) and (3.49) that

$$
\begin{aligned}
\left\|x_{n+1}-\bar{x}\right\|^{2} & \leq\left(1-\epsilon_{n}-\epsilon_{n} \mu \bar{\gamma}\right)^{2}\left\|z_{n}-\bar{x}\right\|^{2}+c_{n} \\
& \leq\left(1-\epsilon_{n}-\epsilon_{n} \mu \bar{\gamma}\right)^{2}\left\|y_{n}-\bar{x}\right\|^{2}+c_{n} \\
& \leq\left(1-\epsilon_{n}-\epsilon_{n} \mu \bar{\gamma}\right)^{2}\left\{\left\|x_{n}-\bar{x}\right\|^{2}-\left\|v_{n}-y_{n}\right\|^{2}+2 t_{n}\left\|v_{n}-y_{n}\right\|\left\|B_{2} v_{n}-B_{2} \bar{x}\right\|\right\}+c_{n}
\end{aligned}
$$


which in turn implies that

$$
\begin{aligned}
\left(1-\epsilon_{n}-\epsilon_{n} \mu \bar{\gamma}\right)^{2}\left\|v_{n}-y_{n}\right\|^{2} \leq & \left(1-\epsilon_{n}-\epsilon_{n} \mu \bar{\gamma}\right)^{2}\left\|x_{n}-\bar{x}\right\|^{2}-\left\|x_{n+1}-x_{n}\right\|^{2} \\
& +2\left(1-t_{n} \epsilon_{n}-\epsilon_{n} \mu \bar{\gamma}\right)^{2}\left\|v_{n}-y_{n}\right\|\left\|B_{2} v_{n}-B_{2} \bar{x}\right\|+c_{n} \\
\leq & \left\|x_{n}-\bar{x}\right\|^{2}-\left\|x_{n+1}-\bar{x}\right\|^{2}+\epsilon_{n}^{2}(1+\mu \bar{\gamma})^{2}\left\|x_{n}-\bar{x}\right\|^{2} \\
& +2 t_{n}\left(1-t_{n} \epsilon_{n}-\epsilon_{n} \mu \bar{\gamma}\right)^{2}\left\|v_{n}-y_{n}\right\|\left\|B_{2} v_{n}-B_{2} \bar{x}\right\|+c_{n} \\
\leq & \left(\left\|x_{n}-\bar{x}\right\|+\left\|x_{n+1}-\bar{x}\right\|\right)\left\|x_{n+1}-x_{n}\right\|+\epsilon_{n}^{2}(1+\mu \bar{\gamma})^{2}\left\|x_{n}-\bar{x}\right\|^{2} \\
& +2 t_{n}\left(1-\epsilon_{n}-\epsilon_{n} \mu \bar{\gamma}\right)^{2}\left\|v_{n}-y_{n}\right\|\left\|B_{2} v_{n}-B_{2} \bar{x}\right\|+c_{n} .
\end{aligned}
$$

Since $\epsilon_{n} \rightarrow 0,\left\|B_{2} v_{n}-B_{2} \bar{x}\right\| \rightarrow 0$ and from (3.32), we obtain that

$$
\lim _{n \rightarrow \infty}\left\|v_{n}-y_{n}\right\|=0
$$

In addition, from the firmly nonexpansivity of $V_{\mu_{n}}^{\left(\Theta_{1}, \varphi_{1}\right)}$, we have

$$
\begin{aligned}
\left\|v_{n}-\bar{x}\right\|^{2}= & \left\|V_{\mu_{n}}^{\left(\Theta_{1}, \varphi_{1}\right)}\left(u_{n}-\mu_{n}\left(\Psi_{1}+\Phi_{1}\right) u_{n}\right)-V_{\mu_{n}}^{\left(\Theta_{1}, \varphi_{1}\right)}\left(\bar{x}-\mu_{n}\left(\Psi_{1}+\Phi_{1}\right) \bar{x}\right)\right\|^{2} \\
\leq & \left\langle\left(u_{n}-\mu_{n}\left(\Psi_{1}+\Phi_{1}\right) u_{n}\right)-\left(\bar{x}-\mu_{n}\left(\Psi_{1}+\Phi_{1}\right) \bar{x}\right), v_{n}-\bar{x}\right\rangle \\
= & \frac{1}{2}\left(\left\|\left(u_{n}-\mu_{n}\left(\Psi_{1}+\Phi_{1}\right) u_{n}\right)-\left(\bar{x}-\mu_{n}\left(\Psi_{1}+\Phi_{1}\right) \bar{x}\right)\right\|^{2}+\left\|v_{n}-\bar{x}\right\|^{2}\right. \\
& \left.\quad-\left\|\left(u_{n}-\mu_{n}\left(\Psi_{1}+\Phi_{1}\right) u_{n}\right)-\left(\bar{x}-\mu_{n}\left(\Psi_{1}+\Phi_{1}\right) \bar{x}\right)-\left(v_{n}-\bar{x}\right)\right\|^{2}\right) \\
\leq & \frac{1}{2}\left(\left\|u_{n}-\bar{x}\right\|^{2}+\left\|v_{n}-\bar{x}\right\|^{2}-\left\|\left(u_{n}-v_{n}\right)-\mu_{n}\left(\left(\Psi_{1}+\Phi_{1}\right) u_{n}-\left(\Psi_{1}+\Phi_{1}\right) \bar{x}\right)\right\|^{2}\right) \\
= & \frac{1}{2}\left(\left\|u_{n}-\bar{x}\right\|^{2}+\left\|v_{n}-\bar{x}\right\|^{2}-\left\|u_{n}-v_{n}\right\|^{2}+2 \mu_{n}\left\langle u_{n}-v_{n},\left(\Psi_{1}+\Phi_{1}\right) u_{n}-\left(\Psi_{1}+\Phi_{1}\right) \bar{x}\right\rangle\right. \\
& \left.\quad-\mu_{n}^{2}\left\|\left(\Psi_{1}+\Phi_{1}\right) u_{n}-\left(\Psi_{1}+\Phi_{1}\right) \bar{x}\right\|^{2}\right),
\end{aligned}
$$

which in turn implies that

$$
\begin{aligned}
\left\|v_{n}-\bar{x}\right\|^{2} \leq & \left\|u_{n}-\bar{x}\right\|^{2}-\left\|u_{n}-v_{n}\right\|^{2}+2 \mu_{n}\left\langle u_{n}-v_{n}\left(\Psi_{1}+\Phi_{1}\right) u_{n}-\left(\Psi_{1}+\Phi_{1}\right) \bar{x}\right\rangle \\
& -\mu_{n}^{2}\left\|\left(\Psi_{1}+\Phi_{1}\right) u_{n}-\left(\Psi_{1}+\Phi_{1}\right) \bar{x}\right\|^{2} \\
\leq & \left\|x_{n}-\bar{x}\right\|^{2}-\left\|u_{n}-v_{n}\right\|^{2}+2 \mu_{n}\left\|u_{n}-v_{n}\right\|\left\|\left(\Psi_{1}+\Phi_{1}\right) u_{n}-\left(\Psi_{1}+\Phi_{1}\right) \bar{x}\right\| .
\end{aligned}
$$


It follows from (3.38) and (3.54) that

$$
\begin{aligned}
& \left\|x_{n+1}-\bar{x}\right\|^{2} \\
& \quad \leq\left(1-\epsilon_{n}-\epsilon_{n} \mu \bar{\gamma}\right)^{2}\left\|z_{n}-\bar{x}\right\|^{2}+c_{n} \\
& \quad \leq\left(1-\epsilon_{n}-\epsilon_{n} \mu \bar{\gamma}\right)^{2}\left\|v_{n}-\bar{x}\right\|^{2}+c_{n} \\
& \quad \leq\left(1-\epsilon_{n}-\epsilon_{n} \mu \bar{\gamma}\right)^{2}\left\{\left\|x_{n}-\bar{x}\right\|^{2}-\left\|u_{n}-v_{n}\right\|^{2}+2 \mu_{n}\left\|u_{n}-v_{n}\right\|\left\|\left(\Psi_{1}+\Phi_{1}\right) u_{n}-\left(\Psi_{1}+\Phi_{1}\right) \bar{x}\right\|\right\}+c_{n}
\end{aligned}
$$

which in turn implies that

$$
\begin{aligned}
&\left(1-\epsilon_{n}-\epsilon_{n} \mu \bar{\gamma}\right)^{2}\left\|u_{n}-v_{n}\right\|^{2} \\
& \leq\left(1-\epsilon_{n}-\epsilon_{n} \mu \bar{\gamma}\right)^{2}\left\|x_{n}-\bar{x}\right\|^{2}-\left\|x_{n+1}-\bar{x}\right\|^{2} \\
&+2 \mu_{n}\left(1-\epsilon_{n}-\epsilon_{n} \mu \bar{\gamma}\right)^{2}\left\|u_{n}-v_{n}\right\|\left\|\left(\Psi_{1}+\Phi_{1}\right) u_{n}-\left(\Psi_{1}+\Phi_{1}\right) \bar{x}\right\|+c_{n} \\
& \leq\left\|x_{n}-\bar{x}\right\|^{2}-\left\|x_{n+1}-\bar{x}\right\|^{2}+\epsilon_{n}^{2}(1+\mu \bar{\gamma})^{2}\left\|x_{n}-\bar{x}\right\|^{2} \\
&+2 \mu_{n}\left(1-\epsilon_{n}-\epsilon_{n} \mu \bar{\gamma}\right)^{2}\left\|u_{n}-v_{n}\right\|\left\|\left(\Psi_{1}+\Phi_{1}\right) u_{n}-\left(\Psi_{1}+\Phi_{1}\right) \bar{x}\right\|+c_{n} \\
& \leq\left(\left\|x_{n}-\bar{x}\right\|+\left\|x_{n+1}-\bar{x}\right\|\right)\left\|x_{n+1}-x_{n}\right\|+\epsilon_{n}^{2}(1+\mu \bar{\gamma})^{2}\left\|x_{n}-\bar{x}\right\|^{2} \\
&+2 \mu_{n}\left(1-\epsilon_{n}-\epsilon_{n} \mu \bar{\gamma}\right)^{2}\left\|u_{n}-v_{n}\right\|\left\|\left(\Psi_{1}+\Phi_{1}\right) u_{n}-\left(\Psi_{1}+\Phi_{1}\right) \bar{x}\right\|+c_{n}
\end{aligned}
$$

Since $\epsilon_{n} \rightarrow 0,\left\|\Psi_{1} u_{n}-\Psi_{1} \bar{x}\right\| \rightarrow 0,\left\|\Phi_{1} u_{n}-\Phi_{1} \bar{x}\right\| \rightarrow 0$ and from (3.32), we obtain that

$$
\lim _{n \rightarrow \infty}\left\|u_{n}-v_{n}\right\|=0
$$

In a similar way, we can get

$$
\left\|u_{n}-\bar{x}\right\|^{2} \leq\left\|x_{n}-\bar{x}\right\|^{2}-\left\|x_{n}-u_{n}\right\|^{2}+2 r_{n}\left\|x_{n}-u_{n}\right\|\left\|\left(\Psi_{2}+\Phi_{2}\right) x_{n}-\left(\Psi_{2}+\Phi_{2}\right) \bar{x}\right\| .
$$

It follows from (3.38) and (3.58) that

$$
\begin{aligned}
\| & x_{n+1}-\bar{x} \|^{2} \\
& \leq\left(1-\epsilon_{n}-\epsilon_{n} \mu \bar{\gamma}\right)^{2}\left\|z_{n}-\bar{x}\right\|^{2}+c_{n} \\
& \leq\left(1-\epsilon_{n}-\epsilon_{n} \mu \bar{\gamma}\right)^{2}\left\|u_{n}-\bar{x}\right\|^{2}+c_{n} \\
& \leq\left(1-\epsilon_{n}-\epsilon_{n} \mu \bar{\gamma}\right)^{2}\left\{\left\|x_{n}-\bar{x}\right\|^{2}-\left\|x_{n}-u_{n}\right\|^{2}+2 r_{n}\left\|x_{n}-u_{n}\right\|\left\|\left(\Psi_{2}+\Phi_{2}\right) x_{n}-\left(\Psi_{2}+\Phi_{2}\right) \bar{x}\right\|\right\}+c_{n}
\end{aligned}
$$


which in turn implies that

$$
\begin{aligned}
&\left(1-\epsilon_{n}-\epsilon_{n} \mu \bar{\gamma}\right)^{2}\left\|x_{n}-u_{n}\right\|^{2} \\
& \leq\left(1-\epsilon_{n}-\epsilon_{n} \mu \bar{\gamma}\right)^{2}\left\|x_{n}-\bar{x}\right\|^{2}-\left\|x_{n+1}-\bar{x}\right\|^{2} \\
&+2 r_{n}\left(1-\epsilon_{n}-\epsilon_{n} \mu \bar{\gamma}\right)^{2}\left\|x_{n}-u_{n}\right\|\left\|\left(\Psi_{2}+\Phi_{2}\right) x_{n}-\left(\Psi_{2}+\Phi_{2}\right) \bar{x}\right\|+c_{n} \\
& \leq\left\|x_{n}-\bar{x}\right\|^{2}-\left\|x_{n+1}-\bar{x}\right\|^{2}+\epsilon_{n}^{2}(1+\mu \bar{\gamma})^{2}\left\|x_{n}-\bar{x}\right\|^{2} \\
&+2 r_{n}\left(1-\epsilon_{n}-\epsilon_{n} \mu \bar{\gamma}\right)^{2}\left\|x_{n}-u_{n}\right\|\left\|\left(\Psi_{2}+\Phi_{2}\right) x_{n}-\left(\Psi_{2}+\Phi_{2}\right) \bar{x}\right\|+c_{n} \\
& \leq\left(\left\|x_{n}-\bar{x}\right\|+\left\|x_{n+1}-\bar{x}\right\|\right)\left\|x_{n+1}-x_{n}\right\|+\epsilon_{n}^{2}(1+\mu \bar{\gamma})^{2}\left\|x_{n}-\bar{x}\right\|^{2} \\
&+2 r_{n}\left(1-\epsilon_{n}-\epsilon_{n} \mu \bar{\gamma}\right)^{2}\left\|x_{n}-u_{n}\right\|\left\|\left(\Psi_{2}+\Phi_{2}\right) x_{n}-\left(\Psi_{2}+\Phi_{2}\right) \bar{x}\right\|+c_{n} .
\end{aligned}
$$

Since $\epsilon_{n} \rightarrow 0,\left\|\Psi_{2} x_{n}-\Psi_{2} \bar{x}\right\| \rightarrow 0,\left\|\Phi_{2} x_{n}-\Phi_{2} \bar{x}\right\| \rightarrow 0$ and from (3.32), we obtain that

$$
\lim _{n \rightarrow \infty}\left\|x_{n}-u_{n}\right\|=0
$$

Notice that

$$
\left\|S_{n} z_{n}-z_{n}\right\| \leq\left\|S_{n} z_{n}-x_{n}\right\|+\left\|x_{n}-u_{n}\right\|+\left\|u_{n}-v_{n}\right\|+\left\|v_{n}-y_{n}\right\|+\left\|y_{n}-z_{n}\right\| .
$$

It follows from (3.35), (3.48), (3.52), (3.57), and (3.61) that

$$
\lim _{n \rightarrow \infty}\left\|S_{n} z_{n}-z_{n}\right\|=0
$$

Moreover, we note that

$$
\begin{aligned}
\left\|S z_{n}-z_{n}\right\| & \leq\left\|S z_{n}-S_{n} z_{n}\right\|+\left\|S_{n} z_{n}-z_{n}\right\| \\
& \leq \sup _{x \in \widetilde{K}}\left\|S x-S_{n} x\right\|+\left\|S_{n} z_{n}-z_{n}\right\|,
\end{aligned}
$$

where $\tilde{K}$ is any bounded subset of $C$. From Lemma 2.2 and (3.63), we obtain that

$$
\lim _{n \rightarrow \infty}\left\|S z_{n}-z_{n}\right\|=0
$$

Next, we show that

$$
\limsup _{n \rightarrow \infty}\left\langle u+\gamma f\left(x^{*}\right)-(I+\mu A) x^{*}, x_{n}-x^{*}\right\rangle \leq 0,
$$


where $x^{*}$ is a solution of optimization problem (3.4). To show this, we choose a subsequence $\left\{z_{n_{j}}\right\}$ of $\left\{z_{n}\right\}$ such that

$$
\begin{aligned}
\limsup _{n \rightarrow \infty}\left\langle u+\gamma f\left(x^{*}\right)-(I+\mu A) x^{*}, z_{n}-x^{*}\right\rangle \\
=\lim _{j \rightarrow \infty}\left\langle u+\gamma f\left(x^{*}\right)-(I+\mu A) x^{*}, z_{n_{j}}-x^{*}\right\rangle .
\end{aligned}
$$

Since $\left\{z_{n}\right\}$ is bounded, there exists a subsequence $\left\{z_{n_{j}}\right\}$ of $\left\{z_{n}\right\}$ such that $z_{n_{j}} \rightarrow \omega$ as $j \rightarrow \infty$. On the other hand, we note that

$$
\left\|x_{n}-z_{n}\right\| \leq\left\|x_{n}-u_{n}\right\|+\left\|u_{n}-v_{n}\right\|+\left\|v_{n}-y_{n}\right\|+\left\|y_{n}-z_{n}\right\|
$$

It follows from (3.48), (3.52), (3.57), and (3.61) that

$$
\lim _{n \rightarrow \infty}\left\|x_{n}-z_{n}\right\|=0
$$

Now, we show that $\omega \in \Omega:=\bigcap_{i=1}^{\infty} F\left(T_{i}\right) \cap \operatorname{GMEP}\left(\Theta_{1}, \varphi_{1}, \Psi_{1}+\Phi_{1}\right) \cap \operatorname{GMEP}\left(\Theta_{2}, \varphi_{2}, \Psi_{2}+\Phi_{2}\right) \cap$ $\left(B_{1}+W_{1}\right)^{-1}(0) \cap\left(B_{2}+W_{2}\right)^{-1}(0)$.

(i) First, we show that $\omega \in \bigcap_{i=1}^{\infty} F\left(T_{i}\right)$. By Lemma 2.2, we have $F(S)=\bigcap_{i=1}^{\infty} F\left(T_{i}\right)$. Suppose the contrary, $\omega \neq S \omega$, from (3.65) and Opial's condition (see [63]), we have

$$
\begin{aligned}
\liminf _{n \rightarrow \infty}\left\|z_{n_{j}}-\omega\right\| & <\liminf _{n \rightarrow \infty}\left\|z_{n_{j}}-S \omega\right\| \\
& \leq \liminf _{n \rightarrow \infty}\left\{\left\|z_{n_{j}}-S z_{n_{j}}\right\|+\left\|S z_{n_{j}}-S \omega\right\|\right\} \\
& \leq \liminf _{n \rightarrow \infty}\left\|z_{n_{j}}-\omega\right\|,
\end{aligned}
$$

which is a contradiction. So, we obtain $\omega \in F(S)=\bigcap_{i=1}^{\infty} F\left(T_{i}\right)$.

(ii) Now, we show that $\omega \in \operatorname{GMEP}\left(\Theta_{1}, \varphi_{1}, \Psi_{1}+\Phi_{1}\right)$. From $v_{n}=V_{\mu_{n}}^{\left(\Theta_{1}, \varphi_{1}\right)}\left(u_{n}-\mu_{n}\left(\Psi_{1}+\right.\right.$ $\left.\left.\Phi_{1}\right) u_{n}\right)$, we know that

$$
\begin{array}{r}
\Theta_{1}\left(v_{n}, y\right)+\varphi_{1}(y)-\varphi_{1}\left(v_{n}\right)+\left\langle\left(\Psi_{1}+\Phi_{1}\right) u_{n}, y-v_{n}\right\rangle \\
+\frac{1}{\mu_{n}}\left\langle K_{1}^{\prime}\left(v_{n}\right)-K_{1}^{\prime}\left(u_{n}\right), y-v_{n}\right\rangle \geq 0, \quad \forall y \in C .
\end{array}
$$

From (H2), we have

$$
\begin{aligned}
& \varphi_{1}(y)-\varphi_{1}\left(v_{n}\right)+\left\langle\left(\Psi_{1}+\Phi_{1}\right) u_{n}, y-v_{n}\right\rangle \\
& \quad+\frac{1}{\mu_{n}}\left\langle K_{1}^{\prime}\left(v_{n}\right)-K_{1}^{\prime}\left(u_{n}\right), y-v_{n}\right\rangle \geq \Theta_{1}\left(y, v_{n}\right), \quad \forall y \in C .
\end{aligned}
$$


Now, replacing $n$ by $n_{j}$ in (3.72), we have

$$
\begin{aligned}
& \varphi_{1}(y)-\varphi_{1}\left(v_{n_{j}}\right)+\left\langle\left(\Psi_{1}+\Phi_{1}\right) u_{n_{j}}, y-v_{n_{j}}\right\rangle \\
& +\frac{1}{\mu_{n_{j}}}\left\langle K_{1}^{\prime}\left(v_{n_{j}}\right)-K_{1}^{\prime}\left(u_{n_{j}}\right), y-v_{n_{j}}\right\rangle \geq \Theta_{1}\left(y, v_{n_{j}}\right), \quad \forall y \in C .
\end{aligned}
$$

Put $v_{t}=t y+(1-t) \omega$ for all $t \in(0,1]$ and $y \in C$. Then, from (3.73), we have

$$
\begin{aligned}
\left\langle v_{t}-\right. & \left.v_{n_{j}},\left(\Psi_{1}+\Phi_{1}\right) v_{t}\right\rangle \\
\geq & \left\langle v_{t}-v_{n_{j}}\left(\Psi_{1}+\Phi_{1}\right) v_{t}\right\rangle-\varphi_{1}\left(v_{t}\right)+\varphi_{1}\left(v_{n_{j}}\right)-\left\langle v_{t}-v_{n_{j}}\left(\Psi_{1}+\Phi_{1}\right) u_{n_{j}}\right\rangle \\
& -\left\langle\frac{K_{1}^{\prime}\left(v_{n_{j}}\right)-K_{1}^{\prime}\left(u_{n_{j}}\right)}{\mu_{n_{j}}}, v_{t}-v_{n_{j}}\right\rangle+\Theta_{1}\left(v_{t}, v_{n_{j}}\right) \\
= & \left\langle v_{t}-v_{n_{j}}\left(\Psi_{1}+\Phi_{1}\right) v_{t}-\left(\Psi_{1}+\Phi_{1}\right) v_{n_{j}}\right\rangle+\left\langle v_{t}-v_{n_{j}},\left(\Psi_{1}+\Phi_{1}\right) v_{n_{j}}-\left(\Psi_{1}+\Phi_{1}\right) u_{n_{j}}\right\rangle \\
& -\varphi_{1}\left(v_{t}\right)+\varphi_{1}\left(v_{n_{j}}\right)-\left\langle\frac{K_{1}^{\prime}\left(v_{n_{j}}\right)-K_{1}^{\prime}\left(u_{n_{j}}\right)}{\mu_{n_{j}}}, v_{t}-v_{n_{j}}\right\rangle+\Theta_{1}\left(v_{t}, v_{n_{j}}\right) .
\end{aligned}
$$

Since $\left\|v_{n_{j}}-u_{n_{j}}\right\| \rightarrow 0$, we have $\left\|\left(\Psi_{1}+\Phi_{1}\right) v_{n_{j}}-\left(\Psi_{1}+\Phi_{1}\right) u_{n_{j}}\right\| \rightarrow 0$ and from Lipschitz continuity of $K_{1}^{\prime}$, we have $\left\|K_{1}^{\prime}\left(v_{n_{j}}\right)-K_{1}^{\prime}\left(u_{n_{j}}\right)\right\| \rightarrow 0$. Further, from the monotonicity of $\Psi_{1}+\Phi_{1}$, we have

$$
\left\langle v_{t}-v_{n_{j}},\left(\Psi_{1}+\Phi_{1}\right) v_{t}-\left(\Psi_{1}+\Phi_{1}\right) v_{n_{j}}\right\rangle \geq 0
$$

Since $x_{n_{j}} \rightarrow \omega,\left\|x_{n_{j}}-u_{n_{j}}\right\| \rightarrow 0$ and $\left\|v_{n_{j}}-u_{n_{j}}\right\| \rightarrow 0$, we have $v_{n_{j}} \rightarrow \omega$. Then, from (H4), (H5), the weakly lower semicontinuous of $\varphi_{1}$, and $\left(K_{1}^{\prime}\left(v_{n_{j}}\right)-K^{\prime}\left(u_{n_{j}}\right)\right) / \mu_{n_{j}} \rightarrow 0$, we obtain that

$$
\left\langle v_{t}-\omega,\left(\Psi_{1}+\Phi_{1}\right) v_{t}\right\rangle \geq-\varphi_{1}\left(v_{t}\right)+\varphi_{1}(\omega)+\Theta_{1}\left(v_{t}, \omega\right), \quad \text { as } j \longrightarrow \infty
$$

From (H1), (H4), and (3.76), we also have

$$
\begin{aligned}
0 & =\Theta_{1}\left(v_{t}, v_{t}\right)+\varphi_{1}\left(v_{t}\right)-\varphi_{1}\left(v_{t}\right) \\
& \leq t \Theta_{1}\left(v_{t}, y\right)+(1-t) \Theta_{1}\left(v_{t}, \omega\right)+t \varphi_{1}(y)+(1-t) \varphi_{1}(\omega)-\varphi_{1}\left(v_{t}\right) \\
& =t\left[\Theta_{1}\left(v_{t}, y\right)+\varphi_{1}(y)-\varphi_{1}\left(v_{t}\right)\right]+(1-t)\left[\Theta_{1}\left(v_{t}, \omega\right)+\varphi_{1}(\omega)-\varphi_{1}\left(v_{t}\right)\right] \\
& \leq t\left[\Theta_{1}\left(v_{t}, y\right)+\varphi_{1}(y)-\varphi_{1}\left(v_{t}\right)\right]+(1-t)\left\langle v_{t}-\omega,\left(\Psi_{1}+\Phi_{1}\right) v_{t}\right\rangle \\
& =t\left[\Theta_{1}\left(v_{t}, y\right)+\varphi_{1}(y)-\varphi_{1}\left(v_{t}\right)\right]+(1-t) t\left\langle y-\omega,\left(\Psi_{1}+\Phi_{1}\right) v_{t}\right\rangle,
\end{aligned}
$$


and hence

$$
0 \leq \Theta_{1}\left(v_{t}, y\right)+\varphi_{1}(y)-\varphi_{1}\left(v_{t}\right)+(1-t)\left\langle y-\omega,\left(\Psi_{1}+\Phi_{1}\right) v_{t}\right\rangle
$$

Letting $t \rightarrow 0$, we have, for all $y \in C$,

$$
0 \leq \Theta_{1}(\omega, y)+\varphi_{1}(y)-\varphi_{1}(\omega)+\left\langle\left(\Psi_{1}+\Phi_{1}\right) \omega, y-\omega\right\rangle
$$

This implies that $\omega \in \operatorname{GMEP}\left(\Theta_{1}, \varphi_{1}, \Psi_{1}+\Phi_{1}\right)$. In a similar way, we can get $\omega \in$ $\operatorname{GMEP}\left(\Theta_{2}, \varphi_{2}, \Psi_{2}+\Phi_{2}\right)$.

(iii) Now, we show that $\omega \in\left(B_{1}+W_{1}\right)^{-1}(0)$. In fact, notice that

$$
y_{n}-s_{n} B_{1} y_{n} \in z_{n}+s_{n} W_{1} z_{n}
$$

Let $\rho \in W_{1} \eta$. Since $W_{1}$ is monotone, we have

$$
\left\langle\frac{y_{n}-z_{n}}{s_{n}}-B_{1} y_{n}-\rho, z_{n}-\eta\right\rangle \geq 0
$$

It follows from the condition (C8) and (3.48) that

$$
\left\langle-B_{1} \omega-\rho, \omega-\eta\right\rangle \geq 0
$$

This implies that $-B_{1} \omega \in W_{1} \omega$, that is $\omega \in\left(B_{1}+W_{1}\right)^{-1}(0)$. In a similar way, we can get $\omega \in$ $\left(B_{2}+W_{2}\right)^{-1}(0)$. Therefore $\omega \in \Omega:=\bigcap_{i=1}^{\infty} F\left(T_{i}\right) \cap \operatorname{GMEP}\left(\Theta_{1}, \varphi_{1}, \Psi_{1}+\Phi_{1}\right) \cap \operatorname{GMEP}\left(\Theta_{2}, \varphi_{2}, \Psi_{2}+\right.$ $\left.\Phi_{2}\right) \cap\left(B_{1}+W_{1}\right)^{-1}(0) \cap\left(B_{2}+W_{2}\right)^{-1}(0)$. Now, from Lemma 2.11, (3.67), and (3.69), we obtain that

$$
\begin{aligned}
& \limsup _{n \rightarrow \infty}\left\langle u+\gamma f\left(x^{*}\right)-(I+\mu A) x^{*}, x_{n}-x^{*}\right\rangle \\
& \quad=\limsup _{n \rightarrow \infty}\left\langle u+\gamma f\left(x^{*}\right)-(I+\mu A) x^{*}, z_{n}-x^{*}\right\rangle \\
& \quad=\lim _{j \rightarrow \infty}\left\langle u+\gamma f\left(x^{*}\right)-(I+\mu A) x^{*}, z_{n_{j}}-x^{*}\right\rangle \\
& \quad=\left\langle u+\gamma f\left(x^{*}\right)-(I+\mu A) x^{*}, \omega-x^{*}\right\rangle \\
& \leq 0 .
\end{aligned}
$$


Finally, we show that $x_{n} \rightarrow x^{*}$ as $n \rightarrow \infty$. Again, from Lemma 2.4 (ii), we compute

$$
\begin{aligned}
&\left\|x_{n+1}-x^{*}\right\|^{2} \\
&=\left\|\epsilon_{n}\left(u+\gamma f\left(S_{n} v_{n}\right)-(I+\mu A) x^{*}\right)+\beta_{n}\left(x_{n}-x^{*}\right)+\left(\left(1-\beta_{n}\right) I-\epsilon_{n}(I+\mu A)\right)\left(S_{n} z_{n}-x^{*}\right)\right\|^{2} \\
& \leq\left\|\left(\left(1-\beta_{n}\right) I-\epsilon_{n}(I+\mu A)\right)\left(S_{n} z_{n}-x^{*}\right)+\beta_{n}\left(x_{n}-x^{*}\right)\right\|^{2} \\
&+2 \epsilon_{n}\left\langle u+\gamma f\left(S_{n} v_{n}\right)-(I+\mu A) x^{*}, x_{n+1}-x^{*}\right\rangle \\
& \leq {\left[\left\|\left(\left(1-\beta_{n}\right) I-\epsilon_{n}(I+\mu A)\right)\left(S_{n} z_{n}-x^{*}\right)\right\|+\beta_{n}\left\|x_{n}-x^{*}\right\|\right]^{2} } \\
&+2 \epsilon_{n} \gamma\left\langle f\left(S_{n} v_{n}\right)-f\left(x^{*}\right), x_{n+1}-x^{*}\right\rangle+2 \epsilon_{n}\left\langle u+\gamma f\left(x^{*}\right)-(I+\mu A) x^{*}, x_{n+1}-x^{*}\right\rangle \\
& \leq {\left[\left(1-\beta_{n}-\epsilon_{n}(1+\mu) \bar{\gamma}\right)\left\|z_{n}-x^{*}\right\|+\beta_{n}\left\|x_{n}-x^{*}\right\|\right]^{2}+2 \epsilon_{n} \gamma \alpha\left\|S_{n} v_{n}-x^{*}\right\|\left\|x_{n+1}-x^{*}\right\| } \\
&+2 \epsilon_{n}\left\langle u+\gamma f\left(x^{*}\right)-(I+\mu A) x^{*}, x_{n+1}-x^{*}\right\rangle \\
& \leq {\left[\left(1-\beta_{n}-\epsilon_{n}(1+\mu) \bar{\gamma}\right)\left\|x_{n}-x^{*}\right\|+\beta_{n}\left\|x_{n}-x^{*}\right\|\right]^{2}+2 \epsilon_{n} \gamma \alpha\left\|x_{n}-x^{*}\right\|\left\|x_{n+1}-x^{*}\right\| } \\
&+2 \epsilon_{n}\left\langle u+\gamma f\left(x^{*}\right)-(I+\mu A) x^{*}, x_{n+1}-x^{*}\right\rangle \\
& \leq\left(1-\epsilon_{n}(1+\mu) \bar{\gamma}\right)^{2}\left\|x_{n}-x^{*}\right\|^{2}+\epsilon_{n} \gamma \alpha\left(\left\|x_{n}-x^{*}\right\|^{2}+\left\|x_{n+1}-x^{*}\right\|^{2}\right) \\
&+2 \epsilon_{n}\left\langle u+\gamma f\left(x^{*}\right)-(I+\mu A) x^{*}, x_{n+1}-x^{*}\right\rangle \\
&=\left(1-2 \epsilon_{n}(1+\mu) \bar{\gamma}+\epsilon_{n}^{2}[(1+\mu) \bar{\gamma}]^{2}+\epsilon_{n} \gamma \alpha\right)\left\|x_{n}-x^{*}\right\|^{2}+\epsilon_{n} \gamma \alpha\left\|x_{n+1}-x^{*}\right\|^{2} \\
&+2 \epsilon_{n}\left\langle u+\gamma f\left(x^{*}\right)-(I+\mu A) x^{*}, x_{n+1}-x^{*}\right\rangle .
\end{aligned}
$$

It follows that

$$
\begin{aligned}
\left\|x_{n+1}-x^{*}\right\|^{2} \leq & \frac{1-2 \epsilon_{n}(1+\mu) \bar{\gamma}+\epsilon_{n}^{2}[(1+\mu) \bar{\gamma}]^{2}+\epsilon_{n} \gamma \alpha}{1-\epsilon_{n} \gamma \alpha}\left\|x_{n}-x^{*}\right\|^{2} \\
& +\frac{2 \epsilon_{n}}{1-\epsilon_{n} \gamma \alpha}\left\langle u+\gamma f\left(x^{*}\right)-(I+\mu A) x^{*}, x_{n+1}-x^{*}\right\rangle \\
= & \frac{1-2 \epsilon_{n}(1+\mu) \bar{\gamma}+\epsilon_{n} \gamma \alpha}{1-\epsilon_{n} \gamma \alpha}\left\|x_{n}-x^{*}\right\|^{2}+\frac{\epsilon_{n}^{2}[(1+\mu) \bar{\gamma}]^{2}}{1-\epsilon_{n} \gamma \alpha}\left\|x_{n}-x^{*}\right\|^{2} \\
& +\frac{2 \epsilon_{n}}{1-\epsilon_{n} \gamma \alpha}\left\langle u+\gamma f\left(x^{*}\right)-(I+\mu A) x^{*}, x_{n+1}-x^{*}\right\rangle \\
\leq & {\left[1-\frac{2((1+\mu) \bar{\gamma}-\gamma \alpha) \epsilon_{n}}{1-\epsilon_{n} \gamma \alpha}\right]\left\|x_{n}-x^{*}\right\|^{2}+\frac{\epsilon_{n}^{2}[(1+\mu) \bar{\gamma}]^{2}}{1-\epsilon_{n} \gamma \alpha} M_{4} } \\
& +\frac{2 \epsilon_{n}}{1-\epsilon_{n} \gamma \alpha}\left\langle u+\gamma f\left(x^{*}\right)-(I+\mu A) x^{*}, x_{n+1}-x^{*}\right\rangle,
\end{aligned}
$$


where $M_{4}=\sup _{n \geq 1}\left\{\left\|x_{n}-x^{*}\right\|^{2}\right\}$. Put $\tau_{n}:=2((1+\mu) \bar{\gamma}-\gamma \alpha) \epsilon_{n} /\left(1-\epsilon_{n} \gamma \alpha\right)$ and

$$
\delta_{n}:=\frac{\epsilon_{n}^{2}[(1+\mu) \bar{\gamma}]^{2}}{1-\epsilon_{n} \gamma \alpha} M+\frac{2 \epsilon_{n}}{1-\epsilon_{n} \gamma \alpha}\left\langle u+\gamma f\left(x^{*}\right)-(I+\mu A) x^{*}, x_{n+1}-x^{*}\right\rangle .
$$

Then, (3.85) reduces to formula

$$
\left\|x_{n+1}-x^{*}\right\|^{2} \leq\left(1-\tau_{n}\right)\left\|x_{n}-x^{*}\right\|^{2}+\delta_{n}
$$

It follows from the condition (C4) and (3.83) that $\sum_{n=1}^{\infty} \tau_{n}=\infty$ and

$$
\begin{aligned}
\limsup _{n \rightarrow \infty} \frac{\delta_{n}}{\tau_{n}}= & \limsup _{n \rightarrow \infty} \frac{1}{2((1+\mu) \bar{\gamma}-\gamma \alpha)} \\
& \times\left[\epsilon_{n}[(1+\mu) \bar{\gamma}]^{2} M_{4}+2\left\langle u+\gamma f\left(x^{*}\right)-(I+\mu A) x^{*}, x_{n+1}-x^{*}\right\rangle\right] \leq 0 .
\end{aligned}
$$

Hence, by Lemma 2.12, we obtain that $x_{n} \rightarrow x^{*}$ as $n \rightarrow \infty$. This completes the proof.

Remark 3.2. The control condition $\lim _{n \rightarrow \infty}\left|\mu_{n+1}-\mu_{n}\right|=0$ and other on (C6)-(C9) are replaced by the strictly weaker conditions: $\lim _{n \rightarrow \infty} \mu_{n} / \mu_{n+1}=1$ as shown in the next example.

Example 3.3. (a) If $\lim _{n \rightarrow \infty}\left|\mu_{n+1}-\mu_{n}\right|=0$, then $\lim _{n \rightarrow \infty} \mu_{n} / \mu_{n+1}=1$.

(b) The converse of $(a)$ is not true.

Proof. (a) Since $0<\mu_{n}<\min \left\{\tilde{\beta}_{1}, \tilde{\gamma}_{1}\right\}$ and $\liminf _{n \rightarrow \infty} \mu_{n}>0$, there exists a constant $\tilde{\mu}$ such that $\mu_{n} \geq \tilde{\mu}>0$ for all $n \in \mathbb{N}$. We observe that

$$
\begin{aligned}
\lim _{n \rightarrow \infty}\left|\frac{\mu_{n}}{\mu_{n+1}}-1\right| & =\lim _{n \rightarrow \infty}\left|\frac{\mu_{n}-\mu_{n+1}}{\mu_{n+1}}\right| \\
& \leq \lim _{n \rightarrow \infty} \frac{\left|\mu_{n+1}-\mu_{n}\right|}{\tilde{\mu}} \\
& =0 .
\end{aligned}
$$

Hence, we obtain that $\lim _{n \rightarrow \infty} \mu_{n} / \mu_{n+1}=1$.

(b) Let $\mu_{n}=((n+1) / n)^{n}$, then $\mu_{n+1}=((n+2) /(n+1))^{n+1}$. We see that

$$
\begin{aligned}
\lim _{n \rightarrow \infty} \frac{\mu_{n}}{\mu_{n+1}} & =\lim _{n \rightarrow \infty}\left(1+\frac{1}{n}\right)^{n} \cdot \frac{(1+1 / n)^{n+1}}{(1+2 / n)^{n+1}} \\
& =e \cdot \frac{e}{e^{2}}=1
\end{aligned}
$$


but

$$
\begin{aligned}
\lim _{n \rightarrow \infty}\left|\mu_{n+1}-\mu_{n}\right| & =\lim _{n \rightarrow \infty}\left|\frac{(1+1 / n)^{n+1}}{(1+2 / n)^{n+1}}-\left(1+\frac{1}{n}\right)^{n}\right| \\
& =e-\frac{1}{e} \\
& \neq 0 .
\end{aligned}
$$

Then, converse of (a) is not true. Hence, (b) is proved.

Remark 3.4. Theorem 3.1 improves and extends [45, Theorem 3.3] in the following respects.

(1) The problem of finding the common element $\bigcap_{i=1}^{\infty} F\left(T_{i}\right) \cap \operatorname{GMEP}\left(\Theta_{1}, \varphi_{1}, \Psi_{1}+\Phi_{1}\right) \cap$ $\operatorname{GMEP}\left(\Theta_{2}, \varphi_{2}, \Psi_{2}+\Phi_{2}\right) \cap\left(B_{1}+W_{1}\right)^{-1}(0) \cap\left(B_{2}+W_{2}\right)^{-1}(0)$ is more general and more complex than the one of finding the common element $F(T) \cap \operatorname{GMEP}\left(\Theta_{1}, \varphi, \Psi_{1}\right) \cap$ $\operatorname{GMEP}\left(\Theta_{2}, \varphi, \Psi_{2}\right) \cap \operatorname{VI}(C, B)$ in [44, Theorem 3.3].

(2) In [44, Algorithm 3.13], the function $K_{i}: C \rightarrow \mathbb{R}$ is chosen as $K_{i}(x)=\|x\|^{2} / 2$ for $i=1,2$.

(3) The conditions (C3) and (C4) of Chantarangsi et al. [44, Theorem 3.3] are replaced by the strictly weaker conditions.

Remark 3.5. Theorem 3.1 improves and extends [45, Theorem 2.1] in the following respects.

(1) Theorem 3.1 extended [64, Theorem 2.1] to finding common element of the set of solutions of a system of generalized mixed equilibrium problem, and the set of infinite family of nonexpansive mapping involves strongly positive linear bounded operator and the optimization problem.

(2) The conditions (C3) and (C4) of Yu and Liang [45, Theorem 2.1] are replaced by the strictly weaker conditions.

\section{Some Applications}

Let $H$ be a real Hilbert space and $g: H \rightarrow(-\infty,+\infty]$ a proper convex lower semicontinuous function. Then, the subdifferential $\partial g$ of $g$ is defined as follows:

$$
\partial g(x)=\{y \in H: g(z) \geq g(x)+\langle z-x, y\rangle, \forall z \in H\}, \quad \forall x \in H
$$

From Rockafellar [65], we know that $\partial g$ is maximal monotone. Let $C$ be a closed and convex subset of $H$, and let $\delta_{C}$ be the indicator function of $C$, that is,

$$
\delta_{C}(x)= \begin{cases}0, & x \in C, \\ +\infty, & x \notin C .\end{cases}
$$

Since $\delta_{C}$ is a proper lower semicontinuous convex function on $H$, the subdifferential $\partial \delta_{C}$ of $\delta_{C}$ is a maximal monotone operator. 
Lemma 4.1 (see [57]). Let $C$ be a nonempty closed convex subset of a real Hilbert space $H$, let $P_{C}$ be the metric projection from $H$ onto $C$, and let $\partial \delta_{C}$ be the subdifferential of $\delta_{C}$, where $\delta_{C}$ is as defined in (4.2) and $J_{r}=\left(I+r \partial \delta_{C}\right)^{-1}$. Then,

$$
y=J_{r} x \Longleftrightarrow y=P_{C} x, \quad \forall x \in H, y \in C .
$$

Now, we consider the existence of solution of the variational inequality (1.6).

Theorem 4.2. Let $C$ be a nonempty closed convex subset of a Hilbert space $H$. Let $\varphi_{1}, \varphi_{2}: C \rightarrow \mathbb{R}$ be two lower semicontinuous and convex functionals, and let $\Theta_{1}, \Theta_{2}: C \times C \rightarrow \mathbb{R}$ be two bifunctions satisfying conditions (H1)-(H5). Let $\Psi_{1}, \Psi_{2}, \Phi_{1}, \Phi_{2}: C \rightarrow H$ be $\tilde{\beta}_{1}$-inverse-strongly monotone mapping, $\tilde{\beta}_{2}$-inverse-strongly monotone mapping, $\tilde{\gamma}_{1}$-inverse-strongly monotone mapping, and $\tilde{\gamma}_{2}$ inverse-strongly monotone mapping, respectively, and let $B_{1}, B_{2}: C \rightarrow H$ be $\tilde{\xi}_{1}$-inverse-strongly monotone mapping and $\tilde{\xi}_{2}$-inverse-strongly monotone mapping, respectively. Let $\left\{T_{i}\right\}_{i=1}^{\infty}: C \rightarrow C$ be an infinite family of nonexpansive mappings, and let $\alpha_{i}=\left(\alpha_{1}^{j}, \alpha_{2}^{j}, \alpha_{3}^{j}\right) \in I \times I \times I$, where $I=[0,1]$, $\alpha_{1}^{j}+\alpha_{2}^{j}+\alpha_{3}^{j}=1, \alpha_{1}^{j}+\alpha_{2}^{j} \leq b<1$, and $\alpha_{1}^{j}, \alpha_{2}^{j}, \alpha_{3}^{j} \in(0,1)$ for all $j=1,2, \ldots$ For all $n \in \mathbb{N}$, let $S_{n}$ and $S$ be $S$-mappings generated by $T_{n}, T_{n-1}, \ldots, T_{1}$ and $\alpha_{n}, \alpha_{n-1}, \ldots, \alpha_{1}$ and $T_{n}, T_{n-1}, \ldots$ and $\alpha_{n}, \alpha_{n-1}, \ldots$, respectively. Assume that

$$
\begin{aligned}
\Omega:= & \bigcap_{i=1}^{\infty} F\left(T_{i}\right) \cap \operatorname{GMEP}\left(\Theta_{1}, \varphi_{1}, \Psi_{1}+\Phi_{1}\right) \\
& \cap \operatorname{GMEP}\left(\Theta_{2}, \varphi_{2}, \Psi_{2}+\Phi_{2}\right) \cap \operatorname{VI}\left(C, B_{1}\right) \cap \operatorname{VI}\left(C, B_{2}\right) \neq \emptyset .
\end{aligned}
$$

Let $f: C \rightarrow H$ be a contraction mapping with a coefficient $\alpha \in(0,1)$, and let $A: C \rightarrow H$ be a strongly positive linear bounded operator with a coefficient $\bar{\gamma} \in(0,1)$. Let $\mu>0$ and $\gamma>0$ be two constants such that $0<\gamma<(1+\mu) \bar{\gamma} / \alpha$. Let $\left\{x_{n}\right\}$ be a sequence defined by $u, x_{1} \in H$ and

$$
\begin{gathered}
u_{n}=V_{r_{n}}^{\left(\Theta_{2}, \varphi_{2}\right)}\left(x_{n}-r_{n}\left(\Psi_{2}+\Phi_{2}\right) x_{n}\right), \\
v_{n}=V_{\mu_{n}}^{\left(\Theta_{1}, \varphi_{1}\right)}\left(u_{n}-\mu_{n}\left(\Psi_{1}+\Phi_{1}\right) u_{n}\right), \\
y_{n}=P_{C}\left(v_{n}-t_{n} B_{2} v_{n}\right), \\
x_{n+1}=\epsilon_{n}\left(u+\gamma f\left(S_{n} v_{n}\right)\right)+\beta_{n} x_{n}+\left(\left(1-\beta_{n}\right) I-\epsilon_{n}(I+\mu A)\right) S_{n} P_{C}\left(y_{n}-S_{n} B_{1} y_{n}\right), \quad \forall n \geq 1,
\end{gathered}
$$

where $\left\{s_{n}\right\} \subset\left(0,2 \tilde{\xi}_{1}\right),\left\{t_{n}\right\} \subset\left(0,2 \tilde{\xi}_{2}\right),\left\{\mu_{n}\right\} \subset\left(0, \min \left\{\tilde{\beta}_{1}, \tilde{\gamma}_{1}\right\}\right),\left\{r_{n}\right\} \subset\left(0, \min \left\{\tilde{\beta}_{2}, \tilde{\gamma}_{2}\right\}\right)$, and $\left\{\epsilon_{n}\right\}$ and $\left\{\beta_{n}\right\} \subset(0,1)$. Assume the following conditions are satisfied:

(C1) for all $i=1,2, K_{i}: C \rightarrow \mathbb{R}$ is strongly convex with constant $\sigma_{i}>0$ and its derivative $K_{i}^{\prime}$ is Lipschitz continuous with constant $\left.v_{i}\right\rangle 0$ such that the function $x \mapsto\left\langle y-x, K_{i}^{\prime}(x)\right\rangle$ is weakly upper semicontinuous for each $y \in C$,

(C2) for all $i=1,2$ and for each $x \in C$, there exist a bounded subset $D_{x} \subset C$ and $z_{x} \in C$ such that, for any $y \notin D_{x}$,

$$
\Theta_{i}\left(y, z_{x}\right)+\varphi_{i}\left(z_{x}\right)-\varphi_{i}(y)+\frac{1}{r_{i}}\left\langle K_{i}^{\prime}(y)-K_{i}^{\prime}(x), z_{x}-y\right\rangle<0,
$$


(C3) $\lim _{n \rightarrow \infty} \alpha_{n}^{1}=0$,

(C4) $\lim _{n \rightarrow \infty} \epsilon_{n}=0$ and $\sum_{n=1}^{\infty} \epsilon_{n}=\infty$,

(C5) $0<\liminf _{n \rightarrow \infty} \beta_{n} \leq \lim \sup _{n \rightarrow \infty} \beta_{n}<1$,

(C6) $0<\liminf _{n \rightarrow \infty} \mu_{n} \leq \lim \sup _{n \rightarrow \infty} \mu_{n}<\min \left\{\tilde{\beta}_{1}, \tilde{\gamma}_{1}\right\}$ and $\lim _{n \rightarrow \infty} \mu_{n} / \mu_{n+1}=1$,

(C7) $0<\liminf _{n \rightarrow \infty} r_{n} \leq \lim \sup _{n \rightarrow \infty} r_{n}<\min \left\{\tilde{\beta}_{2}, \tilde{\gamma}_{2}\right\}$ and $\lim _{n \rightarrow \infty} r_{n} / r_{n+1}=1$,

(C8) $0<\liminf _{n \rightarrow \infty} s_{n} \leq \lim \sup _{n \rightarrow \infty} s_{n}<2 \tilde{\xi}_{1}$ and $\lim _{n \rightarrow \infty} s_{n} / s_{n+1}=1$,

(C9) $0<\liminf _{n \rightarrow \infty} t_{n} \leq \lim \sup _{n \rightarrow \infty} t_{n}<2 \tilde{\xi}_{2}$ and $\lim _{n \rightarrow \infty} t_{n} / t_{n+1}=1$.

Then, the sequence $\left\{x_{n}\right\}$ defined by (4.5) converges strongly to $x^{*} \in \Omega$, provided $V_{r, n}^{(\Theta, \varphi)}$ is firmly nonexpansive, where $x^{*}$ solves the following optimization problem:

$$
\min _{x \in \Omega} \frac{\mu}{2}\langle A x, x\rangle+\frac{1}{2}\|x-u\|^{2}-h(x)
$$

Proof. Now, we show that $\operatorname{VI}\left(C, B_{1}\right)=\left(B_{1}+\partial \delta_{C}\right)^{-1}(0)$ and $\operatorname{VI}\left(C, B_{2}\right)=\left(B_{2}+\partial \delta_{C}\right)^{-1}(0)$, respectively. Set $W_{1}=W_{2}=\partial \delta_{C}$ in Theorem 3.1. Notice that

$$
\begin{aligned}
x^{*} \in\left(B_{1}+\partial \delta_{C}\right)^{-1}(0) & \Longleftrightarrow 0 \in B_{1} x^{*}+\partial \delta_{C} x^{*} \\
& \Longleftrightarrow-B_{1} x^{*} \in \partial \delta_{C} x^{*} \\
& \Longleftrightarrow\left\langle B_{1} x^{*}, y-x^{*}\right\rangle \geq 0 \\
& \Longleftrightarrow x^{*} \in \operatorname{VI}\left(C, B_{1}\right) .
\end{aligned}
$$

In a similar way, we can get

$$
x^{*} \in\left(B_{2}+\partial \delta_{C}\right)^{-1}(0) \Longleftrightarrow x^{*} \in \mathrm{VI}\left(C, B_{2}\right)
$$

From Lemma 4.1, we can conclude the desired conclusion immediately. This completes the proof.

Let $\widehat{T}: C \rightarrow C$ be $k$-strict pseudocontraction mapping. Setting $B=I-\widehat{T}$, we see that, for all $x, y \in C$,

$$
\|(I-B) x-(I-B) y\|^{2} \leq\|x-y\|^{2}+k\|B x-B y\|^{2} .
$$

On the other hand, we note that

$$
\|(I-B) x-(I-B) y\|^{2} \leq\|x-y\|^{2}-2\langle B x-B y, x-y\rangle+\|B x-B y\|^{2} .
$$


For all $x, y \in C$, we obtain that

$$
\langle B x-B y, x-y\rangle \geq \frac{1-k}{2}\|B x-B y\|^{2} .
$$

Then, $B$ is $((1-k) / 2)$-inverse-strongly monotone mapping.

Theorem 4.3. Let $C$ be a nonempty closed convex subset of a Hilbert space $H$. Let $\varphi_{1}, \varphi_{2}: C \rightarrow \mathbb{R}$ be two lower semicontinuous and convex functionals, and let $\Theta_{1}, \Theta_{2}: C \times C \rightarrow \mathbb{R}$ be two bifunctions satisfying conditions (H1)-(H5). Let $\Psi_{1}, \Psi_{2}, \Phi_{1}, \Phi_{2}: C \rightarrow H$ be $\widetilde{\beta}_{1}$-inverse-strongly monotone mapping, $\tilde{\beta}_{2}$-inverse-strongly monotone mapping, $\widetilde{\gamma}_{1}$-inverse-strongly monotone mapping, and $\tilde{\gamma}_{2}-$ inverse-strongly monotone mapping, respectively, and let $B_{1}, B_{2}: C \rightarrow H$ be $\tilde{\xi}_{1}$-inverse-strongly monotone mapping and $\tilde{\xi}_{2}$-inverse-strongly monotone mapping, respectively. Let $\left\{T_{i}\right\}_{i=1}^{\infty}: C \rightarrow C$ be an infinite family of nonexpansive mappings, and let $\alpha_{i}=\left(\alpha_{1}^{j}, \alpha_{2}^{j}, \alpha_{3}^{j}\right) \in I \times I \times I$, where $I=[0,1]$, $\alpha_{1}^{j}+\alpha_{2}^{j}+\alpha_{3}^{j}=1, \alpha_{1}^{j}+\alpha_{2}^{j} \leq b<1$, and $\alpha_{1}^{j}, \alpha_{2}^{j}, \alpha_{3}^{j} \in(0,1)$ for all $j=1,2, \ldots$. For all $n \in \mathbb{N}$, let $S_{n}$ and $S$ be $S$-mappings generated by $T_{n}, T_{n-1}, \ldots, T_{1}$ and $\alpha_{n}, \alpha_{n-1}, \ldots, \alpha_{1}$ and $T_{n}, T_{n-1}, \ldots$ and $\alpha_{n}, \alpha_{n-1}, \ldots$, respectively. Let $\widehat{T}_{i}: C \rightarrow C$ be a $k_{i}$-pseudocontraction mapping for all $i=1,2$. Assume that

$$
\Omega:=\bigcap_{i=1}^{\infty} F\left(T_{i}\right) \cap \operatorname{GMEP}\left(\Theta_{1}, \varphi_{1}, \Psi_{1}+\Phi_{1}\right) \cap \operatorname{GMEP}\left(\Theta_{2}, \varphi_{2}, \Psi_{2}+\Phi_{2}\right) \cap F\left(\widehat{T}_{1}\right) \cap F\left(\widehat{T}_{2}\right) \neq \emptyset
$$

Let $f: C \rightarrow H$ be a contraction mapping with a coefficient $\alpha \in(0,1)$, and let $A: C \rightarrow H$ be a strongly positive linear bounded operator with a coefficient $\bar{\gamma} \in(0,1)$. Let $\mu>0$ and $\gamma>0$ be two constants such that $0<\gamma<(1+\mu) \bar{\gamma} / \alpha$. Let $\left\{x_{n}\right\}$ be a sequence defined by $u, x_{1} \in H$ and

$$
\begin{aligned}
u_{n}= & V_{r_{n}}^{\left(\Theta_{2}, \varphi_{2}\right)}\left(x_{n}-r_{n}\left(\Psi_{2}+\Phi_{2}\right) x_{n}\right), \\
v_{n}= & V_{\mu_{n}}^{\left(\Theta_{1}, \varphi_{1}\right)}\left(u_{n}-\mu_{n}\left(\Psi_{1}+\Phi_{1}\right) u_{n}\right), \\
y_{n}= & \left(1-t_{n}\right) v_{n}+t_{n} \widehat{T}_{2} v_{n}, \\
x_{n+1}= & \epsilon_{n}\left(u+\gamma f\left(S_{n} v_{n}\right)\right)+\beta_{n} x_{n}+\left(\left(1-\beta_{n}\right) I-\epsilon_{n}(I+\mu A)\right) \\
& \times S_{n}\left[\left(1-s_{n}\right) y_{n}+s_{n} \widehat{T}_{1} y_{n}\right], \quad \forall n \geq 1,
\end{aligned}
$$

where $\left\{s_{n}\right\} \subset\left(0,2 \tilde{\xi}_{1}\right),\left\{t_{n}\right\} \subset\left(0,2 \tilde{\xi}_{2}\right),\left\{\mu_{n}\right\} \subset\left(0, \min \left\{\tilde{\beta}_{1}, \tilde{\gamma}_{1}\right\}\right),\left\{r_{n}\right\} \subset\left(0, \min \left\{\tilde{\beta}_{2}, \tilde{\gamma}_{2}\right\}\right)$, and $\left\{\epsilon_{n}\right\}$ and $\left\{\beta_{n}\right\} \subset(0,1)$. Assume the conditions (C1)- (C9) in Theorem 4.2. Then, the sequence $\left\{x_{n}\right\}$ defined by (4.14) converges strongly to $x^{*} \in \Omega:=\bigcap_{i=1}^{\infty} F\left(T_{i}\right) \cap \operatorname{GMEP}\left(\Theta_{1}, \varphi_{1}, \Psi_{1}+\Phi_{1}\right) \cap$ $\operatorname{GMEP}\left(\Theta_{2}, \varphi_{2}, \Psi_{2}+\Phi_{2}\right) \cap F\left(\widehat{T}_{1}\right) \cap F\left(\widehat{T}_{2}\right)$, provided $V_{r, n}^{(\Theta, \varphi)}$ is firmly nonexpansive, where $x^{*}$ solves the following optimization problem:

$$
\min _{x \in \Omega} \frac{\mu}{2}\langle A x, x\rangle+\frac{1}{2}\|x-u\|^{2}-h(x)
$$


Proof. Taking $B_{i}=I-\widehat{T}_{\mathrm{i}}: C \rightarrow H$, we see that $B_{i}: C \rightarrow H$ is $\lambda_{i}$-strict pseudocontraction mapping with $\lambda_{i}=\left(1-k_{i}\right) / 2$ and $F\left(\widehat{T}_{i}\right)=\operatorname{VI}\left(C, B_{i}\right)$ for $i=1$, 2. From Theorem 4.2 , we can conclude the desired conclusion easily. This completes the proof.

\section{Acknowledgments}

This research was supported by the Center of Excellence in Mathematics, the Commission on Higher Education, Thailand (under the Project no. RG-1-53-03-2). P. Sunthrayuth was partially supported by the Centre of Excellence in Mathematics for the Ph.D. Program at KMUTT. Moreover, the authors are greatly indebted to the reviewers for their extremely constructive comments and valuable suggestions leading to the revised version.

\section{References}

[1] J.-W. Peng and J.-C. Yao, "A new hybrid-extragradient method for generalized mixed equilibrium problems, fixed point problems and variational inequality problems," Taiwanese Journal of Mathematics, vol. 12, no. 6, pp. 1401-1432, 2008.

[2] L.-C. Ceng and J.-C. Yao, "A hybrid iterative scheme for mixed equilibrium problems and fixed point problems," Journal of Computational and Applied Mathematics, vol. 214, no. 1, pp. 186-201, 2008.

[3] S. Takahashi and W. Takahashi, "Strong convergence theorem for a generalized equilibrium problem and a nonexpansive mapping in a Hilbert space," Nonlinear Analysis. Theory, Methods $\mathcal{E}$ Applications, vol. 69 , no. 3, pp. 1025-1033, 2008.

[4] E. Blum and W. Oettli, "From optimization and variational inequalities to equilibrium problems," The Mathematics Student, vol. 63, no. 1-4, pp. 123-145, 1994.

[5] C. Jaiboon and P. Kumam, "A general iterative method for addressing mixed equilibrium problems and optimization problems," Nonlinear Analysis. Theory, Methods E Applications, vol. 73, no. 5, pp. 1180-1202, 2010.

[6] L.-C. Ceng, A. R. Khan, Q. H. Ansari, and J.-C. Yao, “Viscosity approximation methods for strongly positive and monotone operators," Fixed Point Theory, vol. 10, no. 1, pp. 35-72, 2009.

[7] L. C. Ceng, H.-Y. Hu, and M. M. Wong, "Strong and weak convergence theorems for generalized mixed equilibrium problem with perturbation and fixed pointed problem of infinitely many nonexpansive mappings," Taiwanese Journal of Mathematics, vol. 15, no. 3, pp. 1341-1367, 2011.

[8] H.-Y. Hu and L.-C. Ceng, "A general system of generalized nonlinear mixed composite-type equilibria in Hilbert spaces," Taiwanese Journal of Mathematics, vol. 15, no. 3, pp. 927-959, 2011.

[9] A. Moudafi and M. Théra, "Proximal and dynamical approaches to equilibrium problems," in IllPosed Variational Problems and Regularization Techniques, vol. 477 of Lecture Notes in Economics and Mathematical Systems, pp. 187-201, Springer, Berlin, Germany, 1999.

[10] N. Nadezhkina and W. Takahashi, "Weak convergence theorem by an extragradient method for nonexpansive mappings and monotone mappings," Journal of Optimization Theory and Applications, vol. 128, no. 1, pp. 191-201, 2006.

[11] M. A. Noor, "General variational inequalities and nonexpansive mappings," Journal of Mathematical Analysis and Applications, vol. 331, no. 2, pp. 810-822, 2007.

[12] P. Kumam and P. Katchang, "A viscosity of extragradient approximation method for finding equilibrium problems, variational inequalities and fixed point problems for nonexpansive mappings," Nonlinear Analysis. Hybrid Systems, vol. 3, no. 4, pp. 475-486, 2009.

[13] S. Plubtieng and P. Kumam, "Weak convergence theorem for monotone mappings and a countable family of nonexpansive mappings," Journal of Computational and Applied Mathematics, vol. 224, no. 2, pp. 614-621, 2009.

[14] X. Qin, Y. J. Cho, and S. M. Kang, "Convergence theorems of common elements for equilibrium problems and fixed point problems in Banach spaces," Journal of Computational and Applied Mathematics, vol. 225, no. 1, pp. 20-30, 2009.

[15] S. Saewan and P. Kumam, "A hybrid iterative scheme for a maximal monotone operator and two countable families of relatively quasi-nonexpansive mappings for generalized mixed equilibrium and variational inequality problems," Abstract and Applied Analysis, vol. 2010, Article ID 123027, 31 pages, 2010. 
[16] S. Saewan and P. Kumam, "Modified hybrid block iterative algorithm for convex feasibility problems and generalized equilibrium problems for uniformly quasi-ø-asymptotically nonexpansive mappings," Abstract and Applied Analysis, vol. 2010, Article ID 357120, 22 pages, 2010.

[17] S. Saewan and P. Kumam, "A new modified block iterative algorithm for uniformly quasi-ø-asymptotically nonexpansive mappings and a system of generalized mixed equilibrium problems," Fixed Point Theory and Applications, vol. 2011, 35 pages, 2011.

[18] S. Saewan and P. Kumam, "A modified hybrid projection method for solving generalized mixed equilibrium problems and fixed point problems in Banach spaces," Computers and Mathematics with Applications, vol. 62, pp. 1723-1735, 2011.

[19] S. Saewan and P. Kumam, "Strong convergence theorems for countable families of uniformly quasi-øasymptotically nonexpansive mappings and a system of generalized mixed equilibrium problems," Abstract and Applied Analysis, vol. 2011, Article ID 701675, 2011.

[20] S. Saewan and P. Kumam, "The shrinking projection method for solving generalized equilibrium problem and common fixed points for asymptotically quasi-ø-nonexpansive mappings," Fixed Point Theory and Applications, vol. 2011, 9 pages, 2011.

[21] A. Tada and W. Takahashi, "Weak and strong convergence theorems for a nonexpansive mapping and an equilibrium problem," Journal of Optimization Theory and Applications, vol. 133, no. 3, pp. 359-370, 2007.

[22] W. Takahashi and M. Toyoda, "Weak convergence theorems for nonexpansive mappings and monotone mappings," Journal of Optimization Theory and Applications, vol. 118, no. 2, pp. 417-428, 2003.

[23] P. Kumam, W. Kumam, and P. Junlouchai, "Generalized systems of variational inequalities and projection methods for inverse-strongly monotone mappings," Discrete Dynamics in Nature and Society, vol. 2011, Article ID 976505, 2011.

[24] P. Kumam, "A relaxed extragradient approximation method of two inverse-strongly monotone mappings for a general system of variational inequalities, fixed point and equilibrium problems," Bulletin of the Iranian Mathematical Society, vol. 36, no. 1, pp. 227-250, 2010.

[25] P. Kumam and C. Jaiboon, "Approximation of common solutions to system of mixed equilibrium problems, variational inequality problem, and strict pseudo-contractive mappings," Fixed Point Theory and Applications, vol. 2011, Article ID 347204, 30 pages, 2011.

[26] P. Katchang and P. Kumam, "Convergence of iterative algorithm for finding common solution of fixed points and general system of variational inequalities for two accretive operators," Thai Journal of Mathematics, vol. 9, no. 2, pp. 343-360, 2011.

[27] P. Katchang and P. Kumam, "A general iterative method of fixed points for mixed equilibrium problems and variational inclusion problems," Journal of Inequalities and Applications, vol. 2010, Article ID 370197, 25 pages, 2010.

[28] P. Katchang, T. Jitpeera, and P. Kumam, "Strong convergence theorems for solving generalized mixed equilibrium problems and general system of variational inequalities by the hybrid method," Nonlinear Analysis. Hybrid Systems, vol. 4, no. 4, pp. 838-852, 2010.

[29] N. Petrot, R. Wangkeeree, and P. Kumam, "A viscosity approximation method of common solutions for quasi variational inclusion and fixed point problems," Fixed Point Theory, vol. 12, no. 1, pp. 165$178,2011$.

[30] S. Saewan and P. Kumam, "A hybrid iterative scheme for a maximal monotone operator and two countable families of relatively quasi-nonexpansive mappings for generalized mixed equilibrium and variational inequality problems," Abstract and Applied Analysis, vol. 2010, Article ID 123027, 31 pages, 2010.

[31] Y. Yao and J.-C. Yao, “On modified iterative method for nonexpansive mappings and monotone mappings," Applied Mathematics and Computation, vol. 186, no. 2, pp. 1551-1558, 2007.

[32] S. Saewan, P. Kumam, and K. Wattanawitoon, "Convergence theorem based on a new hybrid projection method for finding a common solution of generalized equilibrium and variational inequality problems in Banach spaces," Abstract and Applied Analysis, vol. 2010, Article ID 734126, 25 pages, 2010.

[33] J. Zhao and S. He, "A new iterative method for equilibrium problems and fixed point problems of infinitely nonexpansive mappings and monotone mappings," Applied Mathematics and Computation, vol. 215, no. 2, pp. 670-680, 2009.

[34] Y. Yao, Y. J. Cho, and Y.-C. Liou, "Algorithms of common solutions for variational inclusions, mixed equilibrium problems and fixed point problems," European Journal of Operational Research, vol. 212, no. 2, pp. 242-250, 2011.

[35] Y. Yao and N. Shahzad, "New methods with perturbations for non-expansive mappings in Hilbert spaces," Fixed Point Theory and Applications, vol. 2011, no. 79, 2011. 
[36] Y. Yao and N. Shahzad, "Strong convergence of a proximal point algorithm with general errors," Optimization Letters, vol. 4, pp. 635-641, 2010.

[37] Y. Yao, Y.-C. Liou, and C.-P. Chen, "Algorithms construction for nonexpansive mappings and inversestrongly monotone mappings," Taiwanese Journal of Mathematics, vol. 15, pp. 1979-1998, 2011.

[38] Y. Yao, R. Chen, and Y.-C. Liou, "A unified implicit algorithm for solving the triple-hierarchical constrained optimization problem," Mathematical \& Computer Modelling. In press.

[39] P. Sunthrayuth and P. Kumam, "A general iterative algorithm for the solution of variational inequalities for a nonexpansive semigroup in Banach spaces," Journal of Nonlinear Analysis and Optimization, vol. 1, no. 1, pp. 139-150, 2010.

[40] T. Jitpeera and P. Kumam, “A general iterative algorithm for generalized mixed equilibrium problems and variational inclusions approach to variational inequalities," International Journal of Mathematics and Mathematical Sciences, vol. 2011, Article ID 619813, 25 pages, 2011.

[41] T. Jitpeera and P. Kumam, "An extragradient type method for a system of equilibrium problems, variational inequality problems and fixed points of finitely many nonexpansive mappings," Journal of Nonlinear Analysis and Optimization, vol. 1, no. 1, pp. 71-91, 2010.

[42] T. Jitpeera and P. Kumam, "A composite iterative method for generalized mixed equilibrium problems and variational inequality problems," Journal of Computational Analysis and Applications, vol. 13, no. 2, pp. 345-361, 2011.

[43] T. Jitpeera and P. Kumam, "A new hybrid algorithm for a system of mixed equilibrium problems, fixed point problems for nonexpansive semigroup, and variational inclusion problem," Fixed Point Theory and Applications, Article ID 217407, 27 pages, 2011.

[44] W. Chantarangsi, C. Jaiboon, and P. Kumam, "A viscosity hybrid steepest descent method for generalized mixed equilibrium problems and variational inequalities for relaxed cocoercive mapping in Hilbert spaces," Abstract and Applied Analysis, vol. 2010, Article ID 390972, 39 pages, 2010.

[45] L. Yu and M. Liang, "Convergence of iterative sequences for fixed point and variational inclusion problems," Fixed Point Theory and Applications, vol. 2011, Article ID 368137, 15 pages, 2011.

[46] H. H. Bauschke and J. M. Borwein, "On projection algorithms for solving convex feasibility problems," SIAM Review, vol. 38, no. 3, pp. 367-426, 1996.

[47] P. L. Combettes, "Hilbertian convex feasibility problem: convergence of projection methods," Applied Mathematics and Optimization, vol. 35, no. 3, pp. 311-330, 1997.

[48] F. Deutsch and I. Yamada, "Minimizing certain convex functions over the intersection of the fixed point sets of nonexpansive mappings," Numerical Functional Analysis and Optimization, vol. 19, no. 1-2, pp. 33-56, 1998.

[49] H.-K. Xu, "Iterative algorithms for nonlinear operators," Journal of the London Mathematical Society, vol. 66, no. 1, pp. 240-256, 2002.

[50] T. Jitpeera, U. Witthayarat, and P. Kumam, "Hybrid algorithms of common solutions of generalized mixed equilibrium problems and the common variational inequality problems with applications," Fixed Point Theory and Applications, vol. 2011, Article ID 971479, 28 pages, 2011.

[51] T. Jitpeera and P. Kumam, "Hybrid algorithms for minimization problems over the solutions of generalized mixed equilibrium and variational inclusion problems," Mathematical Problems in Engineering, vol. 2011, Article ID 648617, 26 pages, 2011.

[52] R. Wangkeeree, N. Petrot, P. Kumam, and C. Jaiboon, "Convergence theorem for mixed equilibrium problems and variational inequality problems for relaxed cocoercive mappings," Journal of Computational Analysis and Applications, vol. 13, no. 3, pp. 425-449, 2011.

[53] A. Kangtunyakarn, "A new iterative algorithm for the set of fixed-point problems of nonexpansive mappings and the set of equilibrium problem and variational inequality problem," Abstract and Applied Analysis, vol. 2011, Article ID 562689, 24 pages, 2011.

[54] T. Suzuki, "Strong convergence of Krasnoselskii and Mann's type sequences for one-parameter nonexpansive semigroups without Bochner integrals," Journal of Mathematical Analysis and Applications, vol. 305, no. 1, pp. 227-239, 2005.

[55] W. Takahashi, Nonlinear Functional analysis, Yokohama Publishers, Yokohama, Japan, 2000.

[56] K. Aoyama, Y. Kimura, W. Takahashi, and M. Toyoda, "On a strongly nonexpansive sequence in Hilbert spaces," Journal of Nonlinear and Convex Analysis, vol. 8, no. 3, pp. 471-489, 2007.

[57] S. Takahashi, W. Takahashi, and M. Toyoda, "Strong convergence theorems for maximal monotone operators with nonlinear mappings in Hilbert spaces," Journal of Optimization Theory and Applications, vol. 147, no. 1, pp. 27-41, 2010. 
[58] L. C. Ceng, Q. H. Ansari, and S. Schaible, "Hybrid extragradient-like methods for generalized mixed equilibrium problems, systems of generalized equilibrium problems and optimization problems," Journal of Global Optimization. In press.

[59] L.-C. Ceng and J.-C. Yao, "A relaxed extragradient-like method for a generalized mixed equilibrium problem, a general system of generalized equilibria and a fixed point problem," Nonlinear Analysis. Theory, Methods \& Applications, vol. 72, no. 3-4, pp. 1922-1937, 2010.

[60] J. T. Oden, Qualitative Methods on Nonlinear Mechanics, Prentice Hall, Englewood Cliffs, NJ, USA, 1986.

[61] Y. Yao, M. A. Noor, S. Zainab, and Y.-C. Liou, "Mixed equilibrium problems and optimization problems," Journal of Mathematical Analysis and Applications, vol. 354, no. 1, pp. 319-329, 2009.

[62] J. S. Jung, "Iterative algorithms with some control conditions for quadratic optimizations," Panamerican Mathematical Journal, vol. 16, no. 4, pp. 13-25, 2006.

[63] Z. Opial, "Weak convergence of the sequence of successive approximations for nonexpansive mappings," Bulletin of the American Mathematical Society, vol. 73, pp. 591-597, 1967.

[64] L. Yu and M. Liang, "Convergence of iterative sequences for fixed point and variational inclusion problems," Fixed Point Theory and Applications, Article ID 368137, 15 pages, 2011.

[65] R. T. Rockafellar, "On the maximal monotonicity of subdifferential mappings," Pacific Journal of Mathematics, vol. 33, pp. 209-216, 1970. 


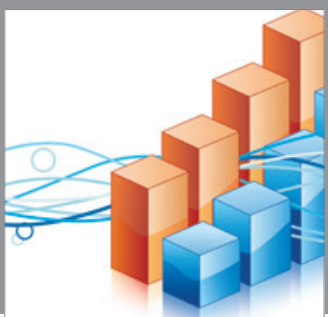

Advances in

Operations Research

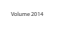

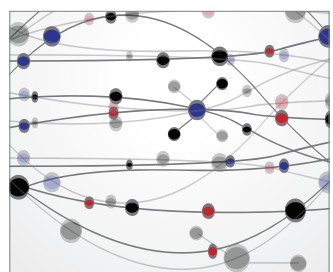

\section{The Scientific} World Journal
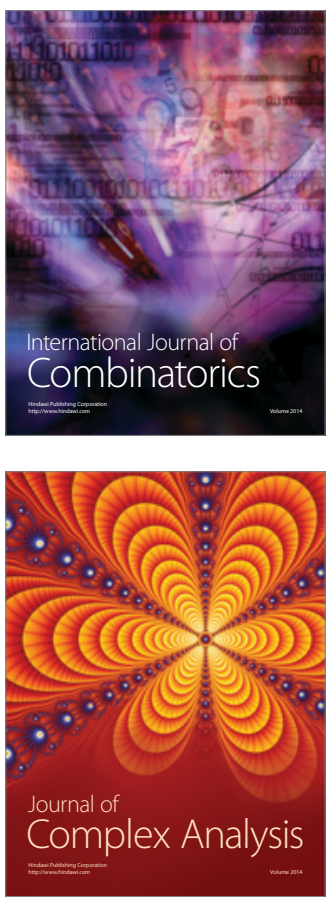

International Journal of

Mathematics and

Mathematical

Sciences
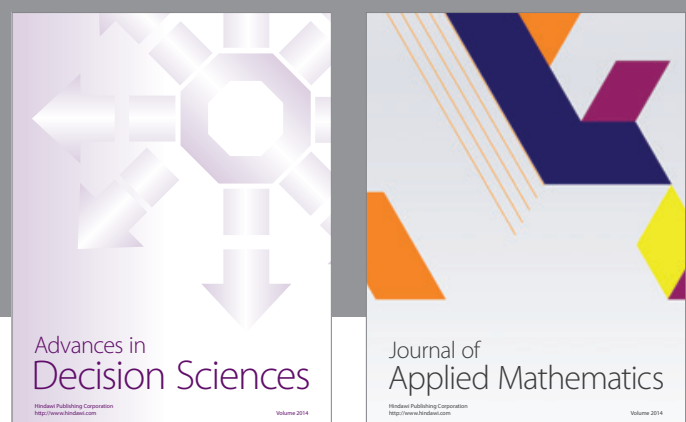

Journal of

Applied Mathematics
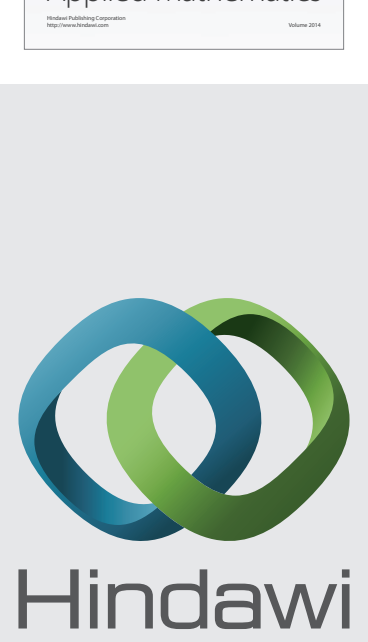

Submit your manuscripts at http://www.hindawi.com
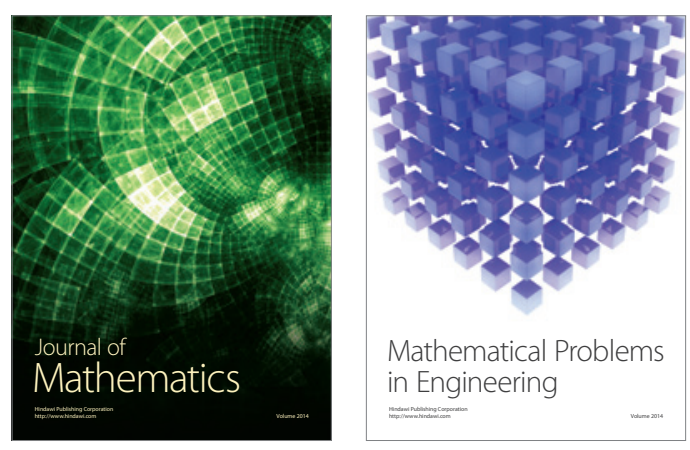

Mathematical Problems in Engineering
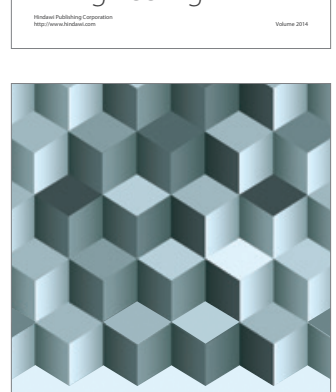

Journal of

Function Spaces
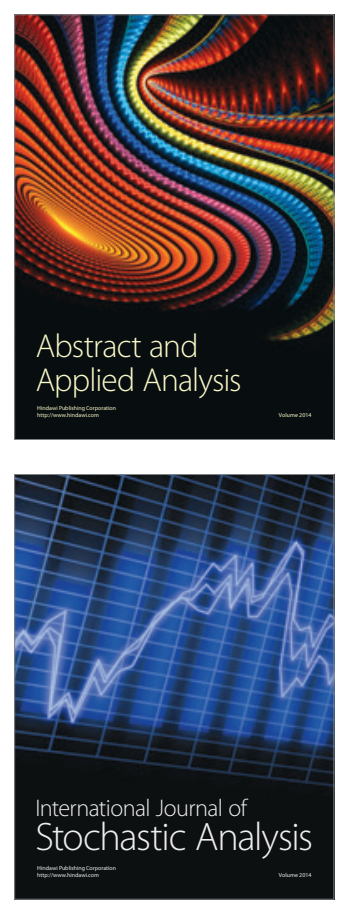

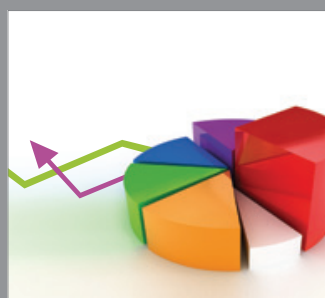

ournal of

Probability and Statistics

Promensencen
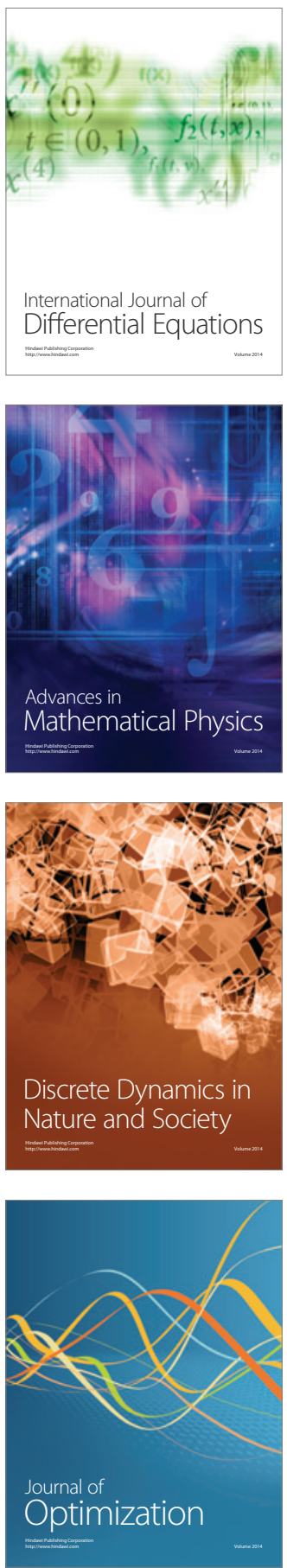